\title{
Fueling growth when oil peaks: directed technological change and the limits to efficiency
}

\author{
Francisco J. André, ${ }^{*}$ \\ Universidad Complutense de Madrid \\ Sjak Smulders, $* *$ \\ Tilburg University, University of Calgary, and CESifo
}

This version: July 19th, 2013

\section{Abstract}

While fossil energy dependency has declined and energy supply has grown in the postwar world economy, future resource scarcity could cast its shadow on world economic growth soon if energy markets are forward looking. We develop an endogenous growth model that reconciles the current aggregate trends in energy use and productivity growth with the intertemporal dynamics of forward looking resource markets. Combining scarcity-rent driven energy supply (in the spirit of Hotelling) with profit-driven Directed Technical Change (in the spirit of Romer/Acemoglu), we generate transitional dynamics that can be qualitatively calibrated to current trends. The long-run properties of the model are studied to examine whether current trends are sustainable. We highlight the role of extraction costs in mining.

Keywords: non-renewable resources, energy, economic growth, innovation, directed technical change.

JEL codes: O41, Q32, Q43.

\footnotetext{
* Dept. of Economic Analysis, Universidad Complutense de Madrid. E-mail: andre@ccee.ucm.es.

** Department of Economics and CentER, Tilburg University. E-mail j.a.smulders@uvt.nl.

This paper has benefited from helpful discussions with Lucas Bretschger, Corrado Di Maria, Clas Eriksson, Matti Liski, Gerard van der Meijden, Pietro Perreto, and Scott Taylor, as well as participants at "Conference on Sustainable Resource Use and Economic Dynamics (SURED)" (Ascona); "First Conference of the Spanish and Portuguese Association of Environmental and Natural Resource Economists (AERNA)" (Vigo, Spain); "XIII Conference of the European Association of Environmental and Resource Economists" (Budapest), "Workshop on Natural Resource Management and Dynamic Optimization" (Girona, Spain); seminars at the University Complutense (Madrid), Helsinki, Montreal and Edmonton. We also thank two anonymous referees for valuable suggestions. Francisco J. André acknowledges financial support from research Projects ECO2012-39553-C04-01 (Spanish Ministry of Science and European Regional Development Fund, ERDF), SEJ 04992 and SEJ-6882 (Andalusian Regional Government).
} 


\section{Introduction}

All developed countries have been heavily dependent on fossil fuels over the past decades, which helps explaining why terrorism and oil security are so high on national policy agendas, why "peak oil" stories (e.g. Deffeyes, 2001) engender emotional discussion, and why national governments postpone signing international climate change treaties. Since fossil fuels are nonrenewable resources, their use cannot grow forever and oil dependence might seem to only become a bigger threat. Energy suppliers can be expected to anticipate future scarcity and manage their extraction-production strategies accordingly. Therefore, one has naturally wondered when and to what degree this dependence puts limits to economic growth.

While in the short run oil shocks can have a big impact on the overall economy, there is little evidence that in the second half of the $20^{\text {th }}$ century oil dependence has caused direct problems for economic growth. According to the trends observed during those years, the supply of energy (both in absolute and in per capita terms) has increased steadily and the share of energy in total cost has declined secularly. Although alternative fuels have become cheaper over time, the use of conventional energy has become more attractive at an even faster pace. Technological change has mainly benefited the productivity of conventional fuels, reducing the need to exploit alternative energy sources. An important indicator of these technological developments is the productivity of energy, as measured by value added per unit of energy, which has steadily increased.

Noticing that the path for the supply of conventional (non-renewable) energy has been increasing but knowing that it will inevitable decline in the future, we can naturally predict a peak in oil supply in the future. The marked slowdown in energy use in the first decade of the $21^{\text {st }}$ century might be the beginning of a change in this direction, but the phenomenon is too recent to know if a structural trend change has occurred already. ${ }^{1}$ As long as oil productivity improves fast enough to offset scarcity, the economic consequences can be small. A key question is therefore how technological developments respond to oil supply changes. Profitseeking entrepreneurs will start looking for energy-saving technologies in the face of energy scarcity. Similarly, forward-looking energy suppliers will adjust their production plans to future events like growing demand because of aggregate economic growth or falling demand because of energy saving. Future resource scarcity could cast its shadow on world economic growth soon when energy and technology markets are forward looking.

\footnotetext{
${ }^{1}$ Per capita energy consumption in USA grew on average at an annual rate $1 \%$ over the period 1949-1999, but over the period 2000-2010 it declined at a rate of 1\% (EIA, 2012).
} 
Our aim is to understand how the trends observed during the second half of the 20th century and the first decade of the 21 st century in aggregate energy use and productivity growth can be linked to future scarcity. We build a model that generate both phases: a first phase of growth fueled by expanding energy supply and a second phase of declining oil production, as part of the same fundamental dynamics that arise from profit-maximizing technology development and energy supply.

The Hotelling (1931) model has been the workhorse model to think about how profitmaximizing forward-looking suppliers of nonrenewable resources -like oil- would allocate production over time. It assumes a finite (cumulative) physical supply and predicts rising prices for extracted resources in the long run. Given that these results carry over to partial and general equilibrium models that assume finite resource supply and forward-looking profit maximizing agents, it seems hard to ignore Hotelling's insights when analyzing growth and resource markets. However, because the model is notoriously difficult to reconcile with stylized facts, the practical application of the insights has been questioned. First, as Gaudet (2007) documents, the empirical pattern of prices is difficult to reconcile with the predictions from the simple partial equilibrium models as long as these models ignore uncertainty and cannot endogenize technology responses to energy scarcity. Second, at the aggregate level, there is a difficulty to reconcile growing energy use with the Hotelling framework. For example, merging the standard neoclassical growth model with the Hotelling model, one typically finds that resource use declines monotonically over time (Dasgupta and Heal, 1974, page 17; see also Benchekroun and Withagen, 2011, who examine the transitional dynamics of the model analytically). In the literature there seems to be a gap between, on the one hand, analytical approaches that start from first principles and apply the Hotelling model but generate extraction patterns that are at odds with facts (e.g. Chakravorty et al., 2008; Van de Ploeg and Withagen, 2011), and, on the other hand, applied and calibrated models that abstract from Hotelling rents for the sake of calibration. For example the DICE model (Nordhaus 1994), the model most often applied to evaluate the consequences of greenhouse emissions associated fossil fuel use, assumes away finiteness of energy supply despite its long time horizon. ${ }^{2}$

\footnotetext{
${ }^{2}$ There are two alternative ways to avoid introducing the Hotelling assumption and results. First, there might be a "backstop technology" that makes the scarce resource input superfluous. Second, the resource supply may be physically unlimited, but subject to increasing extraction costs (Heal, 1976). Both approaches basically assume away the economic relevance of physical scarcity and cannot therefore address its economic consequences. This is not to say that such approaches are not generating useful insights. Rather, we claim that our approach complements these approaches by showing that even with our more "conservative" assumption (i.e. physical finiteness), current trends can be explained. Moreover, physical abundant energy resources, coal in particular,
} 
In this paper we try to fill this gap by developing an analytically tractable model that both can be calibrated to stylized facts and assumes finite resource supply with profitmaximizing intertemporal behavior. By analyzing the key mechanisms of the model, rather than calibrating the model as a black box, we show how the structure of the model allows stylized facts to be replicated. In this procedure, which we dub "qualitative calibration", we analytically derive bounds for parameters we have to pick in each calibration to a model outcome that satisfies a list of well documented stylized facts. This approach allows us to point out that changes in the cost of extraction of the resource play a major role in the calibration. In order to show that this result holds very generally, we deliberately choose to take developments in extraction costs over time as exogenous.

Another contribution of our analytical approach is that we derive the dynamics of our model in terms of a variable that directly captures oil dependence, namely the energy share in GDP. The model predicts a clear-cut relationship between the energy share in GDP, the direction of technological change, and total factor productivity growth. Moreover, given technological change in oil extraction, the dynamics of the energy share can be determined. As a result the model explains how oil dependence - as measured by the energy share in GDP - evolves over time and how it affects the dynamics of technological change and production growth.

When qualitatively calibrating our model, we start from the following stylized facts observed during the last decades of the 20th century: a growing per capita energy supply, declining cost share of energy in GDP, declining energy cost relative to labor cost, and declining energy use per unit of GDP (or, equivalently, increasing energy efficiency), as well as increasing per capita output. We also take into account the evidence of poor substitution between energy and other inputs, and the absence of strong evidence of a predominantly resource-using or resource-saving nature of technological change. The equilibrium dynamics of the model predict that the energy share in GDP first declines and then rises to higher than initial levels. This undershooting phenomenon goes together with fluctuations in total factor productivity (TFP) growth. In the long run, the peak of oil production causes growth rates to fall, partly cushioned by higher rates of TFP growth.

The first building block of the model is the supply of energy, thought of as a nonrenewable resource with a fixed initial endowment. From partial equilibrium resource theory,

cannot perfectly substitute for the physically scarce resources oil and gas (e.g. in transport), so that both types of models are needed. For this reason, when we write "energy", we restrict ourselves mainly to energy from oil and gas rather than from coal. 
it is well known that the price of an exhaustible resource can be decreasing over a time interval if technological change lowers marginal extraction cost rapidly enough (e.g. Krautkraemer, 1998, p. 2068; Berck and Roberts, 1996). The challenge is to include the dynamics of extraction costs in a general equilibrium framework. Tahvonen and Salo (2001) provide such a framework and obtain an inverted U-shaped time pattern for non-renewable resource extraction in a model in which energy can be provided by both a renewable and a non-renewable resource; they consider learning-by-doing but not R\&D. We complement these analyses by focusing on the macroeconomic level: we consider substitution between resource/energy inputs and labor/capital inputs, while technological change not only affects the resources sector, but also the rest of the economy. In our model, the direction of technological change determines whether oil suppliers speed up or slow down depletion over time.

The second building block of our model is the supply of technology. Three types of technological change are distinguished: labor-saving technological change, energy-saving technological change and declines in extraction costs. While the latter are assumed to be exogenous for the reason mentioned above, the first two types of technological change are driven by profit incentives in line with Acemoglu's $(1998,2002)$ theory of endogenous directed technological change. If energy supply grows faster than labor supply, relative energy costs for firms fall (since both inputs are gross complements) and it becomes less profitable to supply resource-saving technologies. However, when the supply of oil has peaked, this pattern reverses. The shifts in the direction of $R \& D$, together with the changes in the scale of the economy as a result from changes in energy supply affect the bias and overall rate of technological change. Existing theoretical papers on growth and non-renewable resources have so far neglected the dynamic interaction between extraction costs and directed technological change. For example, Barbier (1999) and Grimaud and Rougé (2003) assume a unitary elasticity of substitution between inputs so that only one type of technological change can be relevant. In Groth and Schou $(2002,2005)$, growth is driven by capital accumulation rather than knowledge accumulation. The studies by Di Maria and Valente (2008) and Acemoglu et al. (2012) allow for directed technological change in a Hotelling framework but ignore extraction costs. Moreover, the former focuses on the steady state and the latter focuses on the transitional dynamics effects of output (and pollution) taxes, while neither of them attempts to analyse or replicate the empirical facts on energy consumption.

In section 2, we present the final goods production technology and discuss the behavior of firms, consumers, and resource-owners. In section 3 we investigate how the 
model can replicate the stylized facts described above and what is the implied path of technology. Declining extraction costs turn out to be essential to explain the combination of observed stylized facts in the short run. With an increasingly smaller share of extraction costs in total energy cost, declines in extraction costs become increasingly less powerful and we conclude that some of the observed patterns on energy use are unlikely to hold in the long run. In section 4 we study the size and direction of technological change by modeling how firms choose their innovation projects, and we characterize the dynamics of the model. Section 5 presents some discussion on two issues that are not the main focus of our study but provide us with interesting insights and ideas for future research, namely, endogenous extraction costs and policy implications. Section 6 concludes.

\section{Producers, consumers and resource-owners}

A closed economy produces a homogeneous consumption good, using labor and energy services. In turn, labor (energy) services are produced using labor (energy) and a set of specific intermediates. The total supply of labor employed in the final-goods sector, $L$, is a subset of total population, $L^{S}$. The supply of energy results from the endogenous extraction of a non-renewable resource stock. To fix ideas, we will always refer to this input as energy, but a broader interpretation (e.g. materials for production) is possible.

Following Acemoglu (1998), we assume that the productivity of the primary inputs (labor and energy) mainly depends on the quantity and quality of factor-complementary intermediate goods. The quality is a state variable that increases as a result of R\&D effort performed by monopolist firms.

\section{Final goods production}

The final consumption good $(Y)$ is produced using labor services $\left(Y_{L}\right)$ and energy services $\left(Y_{R}\right)$ according to the following CES function with elasticity of substitution equal to $\sigma$ :

$$
Y=A \cdot\left(Y_{L}^{(\sigma-1) / \sigma}+Y_{R}^{(\sigma-1) / \sigma}\right)^{\sigma /(\sigma-1)}
$$

Labor (energy) services are produced according to the following Cobb-Douglas/Romer (1990) production functions: 


$$
\begin{aligned}
& Y_{L}=L^{\beta} \int_{0}^{1} q_{L k} m_{L k}^{1-\beta} d k, \\
& Y_{R}=R^{\beta} \int_{0}^{1} q_{R k} m_{R k}^{1-\beta} d k,
\end{aligned}
$$

where $L$ and $R$ are the primary inputs (labor and energy respectively) employed in the final goods sector, $m_{i k}$ is the use of intermediates of variant $k$ in the production of type $i$ services ( $i$ $=R, L)$ and $q_{i k}$ is the associated quality level. The mass of different intermediates in each sector is normalized to unity.

Final-goods producers take prices as given. Their factor demand (for labor, energy and intermediates) is given by the first-order conditions of the profit maximization problem:

$$
\begin{aligned}
& p_{Y} \frac{\partial Y}{\partial Y_{L}} \frac{\partial Y_{L}}{\partial L}=w_{L} \quad \Leftrightarrow \quad \frac{\beta \theta_{L} p_{Y} Y}{L}=w_{L} \\
& p_{Y} \frac{\partial Y}{\partial Y_{R}} \frac{\partial Y_{R}}{\partial R}=w_{R} \Leftrightarrow \frac{\beta \theta_{R} p_{Y} Y}{R}=w_{R}, \\
& p_{Y} \frac{\partial Y}{\partial Y_{i}} \frac{\partial Y_{i}}{\partial m_{i k}}=p_{m i k} \Leftrightarrow \quad \frac{\theta_{i} p_{Y} Y(1-\beta) q_{i k} m_{i k}{ }^{-\beta}}{\int_{0}^{1} q_{i k} m_{i k}{ }^{1-\beta} d k}=p_{m i k}, \quad i=R, L,
\end{aligned}
$$

where $p_{Y}$ denotes the price of final output, $w_{i}$ is the factor price for primary input $i, p_{\text {mik }}$ is the price of intermediate good $m_{i k}$ and $\theta_{i} \equiv\left(\partial Y / \partial Y_{i}\right) Y_{i} / Y$ is the production elasticity of sector $i$ 's services in final gross output $Y$. From (4) and (5) we see that the share of gross revenue $p_{Y} Y$ that is devoted to remunerate primary inputs equals $\left(\theta_{L}+\theta_{R}\right) \beta$, which equals $\beta$ because of constant returns to scale in production; the remaining share $(1-\beta)$ is spent on intermediates, as can be derived from (6). Hence total factor payments equals $\beta p_{Y} Y$ and $\theta_{i}$ is the share of factor $i$ in factor income. In the sequel we will refer to $\theta_{R}$ as the energy share.

\section{Intermediate goods production and price setting}

The market for intermediates is characterized by monopolistic competition (as in Dixit and Stiglitz 1977). Each producer supplies a unique variety and sets a monopoly price. The cost of producing one unit of $m_{i k}$ at quality $q_{i k}$ is $q_{i k}$ units of the final good. Equation (6) reveals that the elasticity of demand for each intermediate good equals $1 / \beta$. This fact implies that 
monopoly prices for intermediates are set as a mark-up over unit costs $\left(q_{i k} p_{Y}\right)$. As usual, the mark-up is negatively related to the elasticity of demand $1 / \beta$ :

$$
p_{\text {mik }}=q_{i k} p_{Y} /(1-\beta) ; \quad i=R, L
$$

Substituting this price in the demand function (6), we find that all intermediate goods producers within the same sector $i$ produce the same level of output $m_{i}$ :

$$
m_{i k}=m_{i}=\theta_{i} Y(1-\beta)^{2} / Q_{i} ; \quad i=R, L,
$$

where $Q_{L}$ and $Q_{R}$ represent the state of technology as measured by the average quality of labor-related and energy-related inputs, defined as

$$
Q_{i} \equiv \int_{0}^{1} q_{i k} d k \quad i=R, L
$$

\section{Static goods market equilibrium}

The goods market equilibrium can be characterized in terms of primary inputs $(R$ and $L)$ and the state of technology $\left(Q_{L}\right.$ and $\left.Q_{R}\right)$. Substituting equilibrium quantities of intermediate inputs from (8) into the production and demand functions, we first solve for relative factor shares and relative input prices: ${ }^{3}$

$$
\begin{aligned}
& \frac{\theta_{R}}{\theta_{L}}=\left(\frac{Q_{R} R}{Q_{L} L}\right)^{-(1-v) / v}, \\
& \frac{w_{R}}{w_{L}}=\left(\frac{Q_{R}}{Q_{L}}\right)^{-(1-v) / v}\left(\frac{R}{L}\right)^{-1 / v},
\end{aligned}
$$

where $v=1-\beta+\beta \sigma$. Since $\theta_{L}+\theta_{R}=1$, we can solve (10) for each of the factor shares:

3 Differentiating the production function (1) and using the definition $\theta_{i}=\left(\partial Y / \partial Y_{i}\right) Y_{i} / Y$ we find $\theta_{R} / \theta_{L}=\left(Y_{R} / Y_{L}\right)^{1-1 / \sigma}$; using (2), (3) and (8), we get (10). From (4), (5) and (10) we get (11). 


$$
\theta_{R}=1-\theta_{L}=\left[\left(\frac{Q_{R} R}{Q_{L} L}\right)^{(1-v) / v}+1\right]^{-1}
$$

From (11) and (12) we can see that poor substitution ( $\sigma<1$ or, equivalently, $v<1)$ means that an increase in the ratio $Q_{R} / Q_{L}$ implies a fall in relative energy prices and in the energy share. Therefore, an increase in the ratio $Q_{R} / Q_{L}$, which we call the bias of technology, has the interpretation of energy-saving (equivalently, labor-using or labor-biased) technical change. ${ }^{4}$

We next solve for aggregate variables. We express aggregate output as a function of technology and factor inputs by taking into account that the equilibrium level of intermediates use $\left(m_{i}\right)$ depends on output, technology and factor inputs according to (8). In particular, we combine (1), (2), (3), (8), and (12) to arrive at the following expression: ${ }^{5}$

$$
Y=\left(\left(Q_{L} L\right)^{(v-1) / v}+\left(Q_{R} R\right)^{(v-1) / v}\right)^{v /(v-1)}
$$

This equation shows that, in equilibrium, the average quality levels of intermediates use, $Q_{i}$, act as factor augmentation levels. From (13) it is also immediate to interpret $v$ as the elasticity of substitution between factors (measured in effective terms), to be distinguished from the elasticity of substitution between intermediate services $(\sigma)$.

From (13), we can directly write output per worker and per capita income growth as

$$
\begin{aligned}
& Y / L=Q_{L}\left(1-\theta_{R}\right)^{v /(1-v)}, \\
& \hat{Y}-\hat{L}^{S}=\hat{Q}_{L}+\theta_{R}\left(\hat{R}-\hat{L}+\hat{Q}_{R}-\hat{Q}_{L}\right)-\left(\hat{L}^{S}-\hat{L}\right),
\end{aligned}
$$

where hats denote growth rates. Equation (15) shows four sources of growth: changes in the level of (labor-related) technology, growing per capita energy inputs, changes in the bias of technical change and changes in the participation rate. Note also that the second and the third elements are perfect substitutes (regarding growth) and their joint contribution to growth depends on the energy share in output (or production elasticity of energy).

\footnotetext{
${ }^{4}$ The opposite holds if $\sigma>1$, but this is, as will be explained below, not a relevant case.

${ }^{5}$ We have chosen units of $Y$ such that $A=(1-\beta)^{-2(1-\beta)}$, so that the scale constant in (13) becomes unity.
} 
The production of $Y$ serves as intermediates $(m)$ or as final consumption goods $\left(C_{Y}\right)$. Therefore, goods market equilibrium requires $Y=C_{Y}+\int_{0}^{1} q_{L k} m_{L k} d k+\int_{0}^{1} q_{R k} m_{R k} d k$. After substituting (8) and (9), we find that net output $C_{Y}$ is a constant fraction of gross output $Y:^{6}$

$$
C_{Y}=\left[1-(1-\beta)^{2}\right] Y .
$$

\section{Consumer behavior}

The representative consumer maximizes intertemporal utility, specified as $\int_{0}^{\infty} \ln c_{Y} \exp (-\rho t) d t$, subject to his or her budget constraint, where $\rho$ is the utility discount rate, and $c_{Y}$ is per capita consumption of the Y-good. The logarithmic form of the intertemporal utility function implies that the consumer chooses a consumption path along which total spending grows with the difference between the nominal interest rate $r$ and the utility discount rate $\rho$, i.e. $\hat{c}_{Y}+\hat{p}_{Y}=r-\rho$. Using (16) and assuming that population size, $L^{S}$, grows at a constant rate $\hat{L}^{S}$ so that $\hat{c}_{Y}=\hat{C}_{Y}-\hat{L}^{S}=\hat{Y}-\hat{L}^{S}$, we may write the Ramsey rule as

$$
\hat{p}_{Y}+\hat{Y}-\hat{L}^{S}=r-\rho
$$

\section{Natural resource extraction and energy use}

The energy input $R$ is obtained from a non-renewable resource stock $E$ and extraction is costly. Although in the natural resource literature it is common to model extraction cost in terms of output, for the sake of tractability, here we model extraction costs in terms of the resource itself. Specifically, we assume that $1+\mu$ units of the resource stock have to be extracted to produce one unit of energy, where $\mu$ can be interpreted as the unit resource cost to produce energy or, alternatively, as a unit extraction or mining cost. Hence, the resource stock changes over time according to the following differential equation:

$$
\dot{E}=-(1+\mu) R
$$

where a dot over a variable denotes derivation with respect to time. According to (18), of each unit extracted from the resource stock, a fraction $\mu /(1+\mu)$ is lost in the mining process and

\footnotetext{
${ }^{6}$ Note that $p_{Y} C_{Y}=\left[1-(1-\beta)^{2}\right] p_{Y} Y=\beta(2-\beta) p_{Y} Y$ is the value of net output, while $w_{R} R+w_{L} L=\beta p_{Y} Y$ is net factor income (net of intermediates). The excess of net production over net factor payments, $(1-\beta) \beta p_{Y} Y$, is the monopoly rent accruing to intermediate goods suppliers.
} 
only $1 /(1+\mu)$ arrives at the market. This specification is similar to the so-called iceberg costs in trade literature (introduced by Samuelson, 1954). ${ }^{7}$

Our modeling strategy for extraction cost provides us with a very natural way to characterize technological efficiency in the resource extraction sector. Define $Q_{\mu}$ as the amount of energy that can be used in production, $R$, per unit of energy extracted, $(1+\mu) R$, i.e. $Q_{\mu} \equiv 1 /(1+\mu)$ can be interpreted as efficiency in extraction. Accordingly, $\hat{Q}_{\mu}$ is the rate of efficiency improvement in extraction. We will refer to $\hat{Q}_{\mu}$ as improvements in mining efficiency.

We allow unit extraction costs $\mu$ to be decreasing i.e., $\hat{Q}_{\mu}>0$, at least for some period of time. The first interpretation for this assumption is literally taking extraction costs in terms of the resources in the sense that technical improvements allow for better management of well pressure and makes that more can be recovered from a well. Additionally, $E /(1+\mu)$ can be interpreted as economic reserves and then, a decline in $\mu$ can also represent an addition to economic reserves. According to the latter interpretation $\hat{Q}_{\mu}$ encompasses all kinds of extraction cost reductions, including extraction costs at a given mine and discoveries of new reserves (see some additional discussion and references in Section 3).

There is a physical limit to these efficiency improvements, since $\mu$ cannot become negative by definition. In the long-run, the most favorable situation would be one with negligible extraction costs, $\lim _{t \rightarrow \infty} \mu(t)=0$, and with all further improvements exhausted, $\lim _{t \rightarrow \infty} \hat{Q}_{\mu}(t)=0$. In a less favorable situation, extraction costs rise in the long run, possibly due to increasingly difficult geological access to resources. We therefore assume the following:

$$
\lim _{t \rightarrow \infty} \hat{Q}_{\mu}(t) \equiv \lim _{t \rightarrow \infty}\left(\frac{-\dot{\mu}(t)}{1+\mu(t)}\right) \leq 0
$$

The resource owners ${ }^{8}$ decide on the extraction path in order to maximize their discounted profit, $\int_{0}^{\infty} R(t) w_{R}(t) \exp \left[-\int_{0}^{t} r(s) d s\right] d t$, subject to (18). The solution to this problem results in the following modified Hotelling rule and transversality condition:

\footnotetext{
${ }^{7}$ In the partial equilibrium literature, it is common to assume that extraction involves a monetary cost (to pay for the inputs in the mining process) rather than a loss of marketable resources and to assume that extraction costs decrease with the remaining stock of resource (stock effects), and decrease with some technology indicator (technological change). Technically, model extraction costs in terms of resources separates the extraction process from the goods market clearing conditions (if extraction required good $Y$ as an input, the non-arbitrage equation (29) would become more complicated).
} 


$$
\begin{gathered}
r=\hat{w}_{R}+\hat{Q}_{\mu}, \\
\lim _{t \rightarrow \infty} E(t)=0 .
\end{gathered}
$$

Equation (20) is the arbitrage rule for which resource owners are indifferent between, on the one hand, extracting the resource today, selling it and putting the net revenue in the bank at interest rate $r$, and, on the other hand, extracting and selling it later, thus benefiting from higher prices and mining efficiency in the future but forgoing the interest payments. The faster mining efficiency improvements $\left(\hat{Q}_{\mu}\right)$, the more profitable it is to wait extracting, so the slower prices $w_{R}$ have to rise or the higher the interest rate $r$ has to be to make the resource owner indifferent. If technological change in the mining sector is fast enough, such that $0<r$ $<\hat{Q}_{\mu}$, the energy price $w_{R}$ decreases because of the cost reduction effect. However, this situation cannot continue forever: whenever $\mu$ is non-increasing, $\dot{\mu}$ approaches zero in the long run (since $\mu$ is non-negative). Then, as time goes to infinity, equation (20) collapses to the standard Hotelling rule, which implies that, in the long run, the energy price increases at a rate equal to the interest rate. The transversality condition (21) implies that the complete resource stock is depleted asymptotically.

\section{Stylized facts and the forces behind them}

\subsection{Stylized facts}

We now discuss the main stylized facts regarding growth and energy we want to explain in our model. We first list a set of stylized facts derived from U.S. national accounts during the second half of the $20^{\text {th }}$ century (in parentheses we summarize the fact in the notation of the model):

\section{Stylized facts}

1. $\left(\hat{R}>\hat{L}^{S}\right)$ per capita energy use increased at an average annual rate of about 1 percent.

\footnotetext{
${ }^{8}$ We separately discuss consumption and extraction. Since households own the resource stock, the consumption and extraction decision can be integrated. However, this yields the same results, cf. Groth and Schou (2005).
} 
2. $\left(\hat{\theta}_{R}<0\right)$ the share of energy cost in GDP declined at an average annual rate of about 1 percent.

3. $\left(\hat{w}_{R}<\hat{w}_{L}\right)$ energy prices per unit of labor cost declined at an average annual rate of almost 1 percent. $^{9}$

4. $(\hat{Y}>\hat{R})$ energy productivity (GDP per unit of energy input) increased at an annual rate of 1.4 per cent on average. Alternatively, energy intensity declined by 1.4 per cent.

5. $\left(\hat{Y}>\hat{L}^{S}\right)$ per capita income grew at a faster rate than per capita energy use.

The first four stylized facts are reported in Jones (2002, chapter 9) for the US over the period 1950-1998 (based on data from the Energy Information Administration), and confirmed by Sue Wing and Eckaus (2007). Smulders and De Nooij (2003) present qualitatively similar facts for European countries. The fifth stylized fact is the basic feature of postwar growth in the industrialized world (Caselli 2005). Note that this stylized fact is implied by the first and the fourth one.

Fluctuations of energy prices have been large and make it more difficult to establish trends. While we focus on the long-run trend and thus want to average out the energy price spikes around 1973 and 1980, we need to pay attention to the recent years. In Figure 1 we update the figures of Jones (2002) on the energy share. While the trend is declining until 2000 , one could interpret the trend as increasing thereafter. We therefore formulate an alternative for fact 2 :

Stylized fact 2bis: (U-shaped $\theta_{R}(t)$ ) the share of energy cost in GDP has declined for a long period but has started to rise recently.

Two other stylized facts are derived from econometric and other studies that are more sensitive to the approach taken. The first one refers to the direction of technological change. Since technology cannot be observed directly, stylized facts cannot be easily identified for technology trends. Econometric work has provided estimates of the rate and direction of technological change (e.g. by Jorgenson and Fraumendi, 1981; Jorgenson, 1984; Popp, 2002; Sue Wing, 2008; Van der Werf, 2008). Most studies estimate at a sectoral level and find

\footnotetext{
${ }^{9}$ Energy cost relative to wage cost has declined over the period 1948-1998 at an annual average rate of about 0.9 (Jones, 2002, Figure 9.2). However, as is well known there was a big spike between 1973 and 1985.
} 
indications of energy-using technological change in at least some industries. All studies provide evidence of significant effects of disembodied technological change on energy efficiency improvements in a substantial part of the economy. ${ }^{10}$ However, the evidence is mixed with respect to the direction of technical change: in the aggregate, technological change is not strongly biased in either the energy-saving or energy-using direction. To capture this evidence as a stylized fact, we will argue that technical change is close enough to being neutral (or, equivalently, unbiased) over the relevant period and that, by appropriate choice of an initial point in time (denoted by $t=0$ ), technical change can be taken as unbiased initially. $^{11}$

The final fact we deal with refers to substitution elasticities. Studies based on CES production function estimation typically find elasticities of substitution smaller than 1 , but significance and range vary with the exact specification (e.g. Prywes 1986; Kuper and Van Soest, 2002; Van der Werf, 2008; see Neumayer 2003 for a brief survey). In the CGE literature on energy and climate change, the consensus is to assume substitution elasticities not exceeding one (see Van der Werf 2008, Table 1, for an overview).

Summing up, we consider the following two additional stylized facts:

\section{Stylized facts (continued)}

6. $\left(\hat{Q}_{R}(0)=\hat{Q}_{L}(0)\right)$ initially, neutral factor-augmenting technological change.

7. $(v<1)$ poor substitution between energy and other inputs.

\subsection{Qualitative calibration}

We want to check if our analytical model has the right structure to be qualitatively calibrated to the observed stylized facts. By this we mean that, instead of collecting representative data on variables of interest and setting the parameters of the model so as to generate specific values for the endogenous variables that match collected data, we can identify the empirically relevant bounds on the variables of interest and find the corresponding range of parameter values that keep the endogenous variables within the empirically relevant bounds. While the former quantitative calibration procedure is the traditional proven method for employing CGE

\footnotetext{
${ }^{10}$ Sue Wing (2008) aggregates sectoral estimates into a measure of the effect of technical change on aggregate energy intensity. This however yields an estimate of total factor productivity improvements rather than of the bias of technological change. The latter would require aggregating up to the effect of technology on the demand for energy relative to other inputs $(R / L)$ rather than on the energy intensity $(R / Y)$.

${ }^{11}$ As shown below, for our basic results it is not crucial to assume that technological change is exactly neutral, only that it is sufficiently close to neutral. Nevertheless, neutral technical change as a starting point simplifies the calibration exercise considerably.
} 
models in policy evaluation, our qualitative approach is more suited to assess the empirical relevance of our analytical model.

The stylized facts in the previous subsection provide the bounds on the variables of interest. We proceed by deriving the predictions of our model for these variables of interest, after which we determine the bounds on the parameters. In this section we focus on the role of directed technical change and mining efficiency, by identifying the bounds on these variables in particular.

The following lemma summarizes how the key variables of the model depend on the three types of technological change, the discount rate and the evolution of the participation rate, $L / L^{S}$.

Lemma 1. Per capita energy supply, the energy cost share, energy prices relative to wages, energy productivity and output per capita evolve over time according to the following equations:

$$
\begin{aligned}
& \hat{R}-\hat{L}^{s}=n\left(\hat{Q}_{\mu}-\rho\right)-(1-n)\left[\left(\hat{Q}_{R}-\hat{Q}_{L}\right)-\left(\hat{L}-\hat{L}^{s}\right)\right], \\
& \hat{\theta}_{R}=-(1-n)\left[\left(\hat{Q}_{R}-\hat{Q}_{L}\right)+\left(\hat{Q}_{\mu}-\rho\right)-\left(\hat{L}-\hat{L}^{s}\right)\right], \\
& \hat{w}_{R}-\hat{w}_{L}=-\left(\frac{\theta_{R}(1-n)}{1-\theta_{R}}\right)\left(\hat{Q}_{R}-\hat{Q}_{L}\right)-\left(\frac{1-n \theta_{R}}{1-\theta_{R}}\right)\left[\left(\hat{Q}_{\mu}-\rho\right)-\left(\hat{L}-\hat{L}^{s}\right)\right], \\
& \hat{Y}-\hat{R}=\hat{Q}_{R}-n\left(1-\theta_{R}\right)\left[\left(\hat{Q}_{R}-\hat{Q}_{L}\right)+\left(\hat{Q}_{\mu}-\rho\right)-\left(\hat{L}-\hat{L}^{s}\right)\right], \\
& \hat{Y}-\hat{L}^{s}=\hat{Q}_{L}+n \theta_{R}\left[\left(\hat{Q}_{R}-\hat{Q}_{L}\right)+\left(\hat{Q}_{\mu}-\rho\right)\right]+\left(1-n \theta_{R}\right)\left(\hat{L}^{-} \hat{L}^{s}\right),
\end{aligned}
$$

where

$$
n \equiv \frac{v}{v+(1-v)\left(1-\theta_{R}\right)} \in(v, 1) .
$$


In (22)-(26) $n$ can be interpreted as the effective elasticity of substitution between energy and other inputs (notice that for small $\theta_{R}$, as in the data, we have $n \approx v$ ).

According to (22), improvements in mining efficiency favor future extraction over current extraction. Intuitively, resource owners bring the oil to the market when it can be mined relatively more efficiently. A high discount rate $\rho$ has the opposite effect and makes the economy extract now rather than later. Together, $\hat{Q}_{\mu}-\rho$ can be called the "effective postponement stimulus" or "effective patience factor" (cf. Sinclair 1992). The more patient the market effectively is, the more extraction is postponed, which makes it more likely that energy supply will grow rather than decline. Growth in the participation rate, $\hat{L}-\hat{L}^{S}$, also provides an incentive to postpone extraction, since in the future more labor input is available to productively combine with energy. On the other hand, resource-saving technological change in production, as measured by $\hat{Q}_{R}-\hat{Q}_{L}$, reduces energy use over time.

Technological change and discount rates also affect the dynamics of the energy share and the relative energy price. According to (23) and (24), energy-saving technological change as well as mining extraction efficiency improvements makes energy effectively less scarce and thus reduces the energy share and relative energy price, while discounting tends to result in increasing energy share and relative energy price. Again, improving mining efficiency and low discounting make resource owners postpone extraction, which makes energy more abundant and cheaper in the future and reduces the relative price and the share of energy cost in production. The opposite happens with the participation rate: an increasing labor force makes energy scarcer (and thus more expensive) as compared to labor.

Finally, note that mining efficiency improvements and energy technological change play separate roles: with respect to relative energy use and energy prices, mining efficiency improvements and energy-augmented technological change work in opposite directions (cf. (22) and (24)); but for aggregate per capita growth, energy efficiency and energy share, these two types of technological change appear as perfect substitutes (cf. (23), (25) and (26)).

The next step in our qualitative calibration is to find the bounds for the rate of directed technical change that make the model consistent with the three first stylized facts. The following lemma presents these bounds:

\section{Lemma 2 (Energy intensity, energy share and relative energy prices vs. technology bias)}

Stylized facts 1-3 hold at the same time if and only if the bias of technical change is bounded in the following way: 


$$
\max \left\{\hat{L}-\hat{L}^{S}-\hat{Q}_{\mu}+\rho, \frac{\hat{L}-\hat{L}^{S}-\hat{Q}_{\mu}+\rho}{(1-v) \theta_{R}}\right\}<\hat{Q}_{R}-\hat{Q}_{L}<\frac{v}{(1-v) \theta_{L}}\left(\hat{Q}_{\mu}-\rho\right)+\hat{L}-\hat{L}^{S}
$$

Lemma 1 states that technological change must be not too biased in either energy-using or energy-saving direction to replicate the first three stylized facts. The reason is that per capita energy growth and energy share growth both decrease with energy-saving technological change, while the stylized facts require that energy supply and energy share move in opposite directions. The bounds on the rate of directed technical change in Lemma 2 are consistent with the empirical studies and also with our representation of them in stylized fact number 6 (i.e., technical change is initially neutral). As we show in the following proposition (Proposition 1), if we assume neutral technical change (fact 6), then it is immediate to identify necessary and sufficient conditions for all the other stylized facts to hold.

\section{Proposition 1 (short-run matching with stylized facts)}

If fact 6 holds, the following pair of conditions is necessary and sufficient for replicating stylized facts 1- 5:

(i) $\hat{Q}_{\mu}>\rho+\max \left\{\left(\hat{L}-\hat{L}^{S}\right), \frac{-(1-v) \theta_{L}}{v}\left(\hat{L}-\hat{L}^{S}\right)\right\}$,

(ii) $\hat{Q}_{R}>n \theta_{L}\left[\left(\hat{Q}_{\mu}-\rho\right)-\left(\hat{L}-\hat{L}^{S}\right)\right]$.

Note that, under poor substitution (stylized fact 7), condition (i) implies $\hat{Q}_{\mu}>\rho$, since the second term at the RHS is clearly nonnegative. Therefore, Proposition 1 shows that the model can replicate the stylized facts provided extraction costs fall sufficiently fast, so that there is a positive and large enough "postponement stimulus" $\left(\hat{Q}_{\mu}-\rho>0\right)$. Condition (ii) states that energy-augmenting technical change must be large enough. This is needed to ensure that energy efficiency is increasing even though it is getting more intensively used and its share in total income is declining.

The proposition highlights the role of extraction costs. In particular, the trends of declining energy dependence (fact 2) and growing energy supply (fact 1) are to be understood in the model as the result of the physical constraints in extraction of resources becoming less severe (i.e. $\hat{Q}_{\mu}$ positive and sufficiently large). This insight follows directly from the Ramsey rule (17), the Hotelling rule (20) and the demand for energy (equation (5) differentiated with respect to time), which jointly imply the following relationship: 


$$
\hat{R}-\hat{L}^{S}=\hat{\theta}_{R}+\hat{Q}_{\mu}-\rho
$$

To have $\hat{R}-\hat{L}^{S}>0$, fact 1 , and $\hat{\theta}_{R}<0$, fact 2 , we must have $\hat{Q}_{\mu}>\rho$, i.e., improvements in mining efficiency need to be strong enough in the short run. Regarding the plausibility of this assumption, there is quite strong evidence that extraction costs declined during the first part of the 20th century until the beginning of the 80s. Barnett and Morse (1963) concluded that, in the period 1870-1958, although the economy became much more mineral intensive the unit extraction costs for minerals production declined significantly with an increase in the rate of decline after 1920. As a matter of fact, the minerals sector showed a decline in unit cost more than one-half the decline in non-resource sectors. Some years later, Barnett (1979) updated the cost estimates for natural resource industries and the results were essentially the same. According to some later studies, the decline in extraction cost for metals continued clearly in the 1970s (Johnson, Bell, and Bennett 1980, Hall and Hall, 1984). See Krautkraemer (2005) for a summary of this evidence. In the two last decades of the 20th century and the first decade of the $21 \mathrm{st}$, the evidence is mixed and, as time goes by, there is less evidence in favor of decreasing and more evidence pointing to increasing extraction costs, although even in the three last decades there is some evidence on extraction costs reductions. For example, Chermak and Patrick (1995) find a decreasing trend in natural gas extraction during the last part of the 80s. Fagan (1997) reports a reduction in finding costs for oil in the US during the period 1977-1994. EIA (2011) reports a decrease in average worldwide finding costs for the Mayor Energy Producers in the 2007-2009 period.

\section{3. "Peaking oil" and the driving forces in the long run}

Since the total stock of energy resources is non-renewable, the flow of energy supply cannot keep growing and must peak at some instant. The question then is to what extent the current trends in energy supply, energy share, energy price, energy productivity and economic growth can be sustained when oil has peaked and per capita energy growth turn negative. To examine this, we now use the steady state of the model (more specifically, the long run version of equations (22)-(26)). For the moment, we take rates of technological change as given and asymptotically constant. Moreover, we assume that, in the long run, the participation rate in the final-goods sector is constant. 
Equation (23) is then a differential equation in the single variable $\theta_{R}$, and reveals that depending on relative magnitudes of the different types of technical change, the energy share either becomes zero or one, as we state in the following proposition.

Lemma 3 (Solow versus Cake-eating model) Assume $\sigma<1$, constant rates of technological change and constant participation rate $L / L^{S}$ in the long run. Then, in the long run,

(i) if $\hat{Q}_{\mu}+\hat{Q}_{R}-\hat{Q}_{L}>\rho$, only labor contributes to per capita output growth, $\theta_{R}=0$ and $\hat{Y}-\hat{L}^{S}=\hat{Q}_{L}$.

(ii) if $\hat{Q}_{\mu}+\hat{Q}_{R}-\hat{Q}_{L}<\rho$, only energy contributes to per capita output growth, $\theta_{R}=1$ and $\hat{Y}-\hat{L}^{S}=\hat{Q}_{\mu}+\hat{Q}_{R}-\rho$.

(iii) if $\hat{Q}_{\mu}+\hat{Q}_{R}-\hat{Q}_{L}=\rho$, both energy and labor contribute to growth and per capita growth rate equals the rate of labor-augmenting technological change, $0<\theta_{R}<1$ and $\hat{Y}-\hat{L}^{S}=\hat{Q}_{\mu}+\hat{Q}_{R}-\rho=\hat{Q}_{L}$

This lemma shows that, in cases (i) and (ii), the model behaves in the long run as the Solow Neoclassical model and the Dasgupta/Heal (1974, 1979) cake-eating model, respectively. Poor substitution implies that the relatively scarce factor dominates. If the rates of technological change which affect directly energy inputs are relatively large as compared to labor-saving technical change $\left(\hat{Q}_{\mu}+\hat{Q}_{R}-\hat{Q}_{L}>\rho\right)$, energy becomes less and less scarce in effective terms, and the economy is no longer dependent on oil in the long run $\left(\theta_{R}\right.$ tends to $0)$. Then the economy's per capita growth rate equals the rate of labor-augmenting technological change (as in the Solow-model).

On the other hand, with low improvements in energy or mining efficiency, energy becomes the constraining factor, the economy becomes increasingly dependent on oil and the role of labor becomes negligible ( $\theta_{R}$ tends to 1 ). Per capita growth is then determined by resource-related technical change and the discount rate (as in a cake-eating model, Dasgupta and Heal, 1979) ${ }^{12}$. Only if the effects of energy-related technological change on relative energy demand exactly offset those of labor-related technological change, the energy share stays constant over time and both energy and labor keep contributing to output.

\footnotetext{
12 Note, however, that the standard cake-eating model does not distinguish between energy efficiency improvements and mining efficiency improvements, as we do.
} 
We can now find expressions for the rate of change of other key variables in the long run, which allow us to check whether the stylized facts that we listed above can be sustained in the long run. We conclude the following:

Proposition 2 (Long Run) Assume $\sigma<1$. If rates of technological change are constant in the long run with $\lim _{t \rightarrow \infty} \hat{Q}_{\mu}(t) \leq 0, \lim _{t \rightarrow \infty} \hat{Q}_{R}(t) \geq 0$ and $\lim _{t \rightarrow \infty} \hat{Q}_{L}(t) \geq 0$, then, in the long run,

(i) Stylized facts $1\left(\hat{R}>\hat{L}^{S}\right)$ and $3\left(\hat{w}_{R}<\hat{w}_{L}\right)$ cannot be sustained;

(ii) Stylized fact $2\left(\hat{\theta}_{R}<0\right)$ is sustained if and only if $\hat{Q}_{\mu}+\hat{Q}_{R}-\hat{Q}_{L}>\rho$;

(iii) Stylized fact $4(\hat{Y}>\hat{R})$ is sustained unless $\hat{Q}_{R}=0$;

(iv) Stylized fact $5\left(\hat{Y}>\hat{L}^{S}\right)$ is sustained unless $\hat{Q}_{R}+\hat{Q}_{\mu} \leq \rho$.

This proposition describes the long-run situation, allowing for either constant or increasing extraction costs in the long run. The first part reports that per capita energy supply must peak and relative energy prices must rise in the long run (therefore, stylized facts 1 and 3 will get reversed in the future). Growing scarcity of energy inputs tends to fuel increases in energy productivity, because of diminishing returns. Positive energy- and labor-augmenting technological progress adds to this tendency, which supports continuation of stylized fact 4 . However, if energy-related technological change is low, energy inputs become scarcer over time not only in physical terms, but also in effective terms, and the energy share must increase in contrast to what characterizes current short-run dynamics (stylized fact 2). Then energy becomes asymptotically the sole driver of GDP growth, (i.e. $\lim _{t \rightarrow \infty} \theta_{R}=1$, see Lemma 3 ) and only energy-augmenting technical progress can drive energy productivity improvements. If energy-augmenting technological progress is absent, energy productivity becomes asymptotically stagnant (as stated in the third part of the proposition).

For per capita income to keep increasing, we need positive technological progress related to the factor that becomes effectively scarcer over time: if the role of energy vanishes over time (when $\hat{Q}_{\mu}+\hat{Q}_{R}-\rho>\hat{Q}_{L}$ ), labor is the binding factor and positive laboraugmenting technical progress drives per capita growth as in the Solow world; if energy is the binding factor in the long run, energy-augmenting technical progress needs to be large enough to offset the drag on growth because of depletion (as driven by discount rate $\rho$ ) and increases in extraction costs (in particular, we need $\hat{Q}_{R}>\rho-\hat{Q}_{\mu}$ ). 
We conclude from Lemma 3 and Proposition 2 part iv that whether the per capita growth prospects change after oil has peaked depends crucially on which of the two is relatively large: energy-related or labor-related technical change. With such a large role for technology, it becomes crucial to examine the driving forces behind technological change. In the next section we complete the presentation of the model by introducing the mechanism which drives the rate of energy-saving and labor-saving technological change.

\section{The direction of technical change and the dynamics of the model}

In this section we model the economic mechanisms driving factor-augmenting technological change. This will allow us to study the full dynamics of the model and get further insights about the connections between technical change, energy use and economic growth, what provides us with a two-fold value added.

Firstly, note that in Section 3 we have shown how our model can be made consistent with empirical evidence by picking the right path for technical change. The novelty in this section is that, instead of resting on an arbitrarily chosen path for technology, we conclude that it is possible to get, as an endogenous result, a path that is consistent with empirical evidence. In the previous section we discussed how technical change matters for resource extraction. Now we complete the picture by discussing how resource scarcity matters for the evolution of technology. We will find that the knife-edge situation from the third part of Lemma 3 arises as the long-run equilibrium of the model when innovation is explicitly considered.

Secondly, Section 3 shows how making different assumptions on the future evolution of technical change results in different long run forecasts. Now we can make more precise predictions since modeling the economic forces driving technical change also gives us more information about the future behavior of production and resource extraction (see Propositions 3 and 4 below).

\subsection{Modeling endogenous factor-augmenting technological change}

We now model the economic mechanisms driving factor-augmenting technological change by assuming that each intermediate goods producer improves the quality of his or her good by 
investing in-house in research and development activities. We choose the simplest endogenous growth formulation for the investment technology of a single firm: ${ }^{13}$

$$
\dot{q}_{i k}=\frac{Q_{i}}{\kappa_{i}} D_{i k}
$$

where $D_{i k}$ represents the amount of resources (labor) spent on research and development by firm $k$ in sector $i$. Apart from the cost parameter $\kappa_{i}$, the productivity in development activities depends on spillovers. An individual firm builds on the knowledge accumulated in the past by all firms in the sector (see Popp, 2002, for evidence with respect to energy-related research). This knowledge stock is proxied by the current aggregate quality level $Q_{i}$. The firm takes it as given and neglects that its own current development efforts expand the knowledge stock on which future development builds. Thus, intertemporal spillovers arise, which play an important role in preventing the returns to innovation to fall over time. Since production costs rise with the quality level of the product, the return on subsequent innovation tends to fall. However, intertemporal spillovers reduce the cost of innovation, which boosts the rate of return. Under the present specification, both forces exactly offset each other in the long run and rates of return can be sustained.

Each firm $k$ in sector $i$ chooses innovation effort $D_{i k}$ in order to maximize the net present value of its profits. The associated optimal control problem results in the following no-arbitrage equation: ${ }^{14}$

$$
\left(\frac{\beta}{1-\beta}\right) \frac{p_{Y} m_{i} Q_{i}}{w_{D} \kappa_{i}}+\hat{w}_{D}-\hat{Q}_{i} \leq r, \quad \text { with equality if } D_{i k}>0 ; \quad i=R, L
$$

\footnotetext{
${ }^{13}$ Our formulation implies that we end up with a "first generation" endogenous growth model a la Romer (1990) and Grossman and Helpman (1991). In these models a bigger economy grows faster (as per the so-called scale effect). However, we are interested in the evolution of the energy-related variables rather than the effect of the size of the economy, so that the scale effect is not problematic in our case. Following Howitt (1999), we can easily remove the scale effect by assuming that the mass of firms in each sector equals $N$ (rather than one), that $N$ grows at the same rate as population, and that spillovers are related to the average quantity of knowledge per firm. It turns out that most of the equations in this paper go through after redefining $L^{S}$ as being the amount of effective labor supplied per person (rather than the total amount of labor) and $L$ and $D$ as employment in production and research, respectively, as a share of the total workforce. None of the conclusions will be qualitatively affected by this change in modeling. Details are available upon request.

${ }^{14}$ The firm maximizes the present value of profits, $\int\left[p_{m i k} m_{i k}-q_{i k} m_{i k} p_{y}-w_{D} D_{i k}\right] \exp \left[-\int_{0}^{t} r(s) d s\right] d t$, subject to (6) and (28) and using $m_{i k}$ and $D_{i k}$ as control variables, taking as given all prices, aggregate variables and quantities of other firms. See Appendix B for the derivation of equation (29).
} 
where $w_{D}$ is the unit cost of development effort $D_{i k}$ and can be understood as the researcher's wage. Equation (29) states that each firm invests until the marginal net returns from investment equal the cost of capital $r$ and no investment takes place if even at zero investment the cost of capital is higher than the returns. The first term on the left-hand side is the direct return from supplying goods of higher quality. Profits rise with quality in proportion to sales $m_{i}$ and the average quality $Q_{i}$. The term $\hat{w}_{D}-\hat{Q}_{i}$ equals the expected rate of change in the cost of quality improvements. Fast quality growth in the sector (captured by $\hat{Q}_{i}$ ) implies large spillovers and cheaper development in the future, which provides an incentive to postpone innovation and reduces the returns to investment. A higher future cost of development effort (captured by $\hat{w}_{D}$ ) has an opposite effect.

\subsection{The dynamics with endogenous technological change}

We now derive expressions for, first, sectoral differences in technological change, which will amount to the bias of technological change, and, second, measures of aggregate technological change, which will give insight in total factor productivity change. We assume that there is a constant supply of labor, $L^{S}$, which is perfectly mobile across production and research. Hence we have $L+D=L^{S}$, were $D$ is total R\&D effort. We denote total research effort by $D$, which is allocated over the labor and energy sectors:

$$
D=D_{L}+D_{R}
$$

where $D_{i}=\int_{0}^{1} D_{i k} d k$ is aggregate research effort in sector $i(i=L, R)$. We find sector-level technological change by aggregating (28) over all firms $k$ in sector $i$ and substituting (9), which yields:

$$
\hat{Q}_{i}=D_{i} / \kappa_{i} ; \quad i=R, L
$$

This equation clarifies that $\kappa_{i}$ represents the amount of effort needed for each percentage point of growth of average quality $Q_{i}$.

We now choose the normalization $\kappa_{L}+\kappa_{R}=1$, which ensures that our cost parameters $\kappa_{i}$ reflect relative differences in effort requirements only (absolute effort levels will by governed by total labor, cf. equation (39) below). 
Substituting (8) to eliminate $m_{i} Q_{i}$, we write the no-arbitrage expressions in (29) as

$$
r-\hat{w}_{D} \geq \frac{\beta(1-\beta)}{w} \frac{\theta_{i}}{\kappa_{i}}-\hat{Q}_{i}, \quad \text { with equality if } \hat{Q}_{i}=D_{i} / \kappa_{i}>0 ; \quad i=L, R
$$

where $w \equiv w_{D} / p_{Y} Y$ represents the wage, scaled by GDP.

For the moment we will focus on interior solutions (i.e., there is positive R\&D in both sectors); we defer the discussion of corner solutions until we build our phase diagram. Under this assumption, subtracting the equations for $R$ and $L$ in (32) from each other, using $\theta_{L}=1-\theta_{R}$ and $\kappa_{L}+\kappa_{R}=1$, we can derive the following expression which governs the evolution of the bias of technological change:

$$
\hat{Q}_{R}-\hat{Q}_{L}=\frac{\beta(1-\beta)}{w \kappa_{L} \kappa_{R}}\left(\theta_{R}-\kappa_{R}\right)
$$

Equation (33) reveals that the direction of technical change is driven by the energy share, which represents the importance of energy for production. As it was discussed above, under poor substitution, the energy share can be taken as a measure of scarcity (the more scarce energy is, the higher $\theta_{R}$ ). Therefore, equation (33) shows how the direction of technological change responds to resource scarcity. If natural resources turn out to be scarce and hence energy becomes very important for production (i.e. the energy share $\theta_{R}$ is high as compared to the unit cost of energy-complementary $\left.\mathrm{R} \& \mathrm{D}, \kappa_{R}\right)$, then it pays to allocate more resources to the energy sector and technical change will become more energy-saving. The opposite happens when the energy share is low. This is an important difference with respect to an exogenous technical change framework, in which technology do not react to resource scarcity.

To derive implications for aggregate innovation, again for an interior solution, using (30) and (31) we get $D=\kappa_{L} \hat{Q}_{L}+\kappa_{R} \hat{Q}_{R}$. Adding the equations for $R$ and $L$ in (32) after multiplying by $\kappa_{i}$ yields

$$
r-\hat{w}_{D}=\frac{\beta(1-\beta)}{w}-D
$$

Noting that $\hat{w}=\hat{w}_{D}-\hat{Y}-\hat{p}_{Y}$ and substituting (17), we find 


$$
\dot{w}=(D+\rho) w-\beta(1-\beta) .
$$

Since labor is perfectly mobile across production and research, in equilibrium $w_{L}=w_{D}$ must hold. Using this condition together with $L+D=L^{S}$ and (4) we find the following expression for the equilibrium allocation of labor between production and research:

$$
\begin{aligned}
& L=\beta \frac{1-\theta_{R}}{w}, \\
& D=L^{S}-\beta \frac{1-\theta_{R}}{w} .
\end{aligned}
$$

Using (32) under the assumption of interior solution, together with (34) to eliminate $r-\hat{w}_{D}$ and (37) to eliminate $D$, we can derive the amount of research in each sector:

$$
D_{i}=\beta(1-\beta) \frac{\theta_{i}-\kappa_{i}}{w}+\kappa_{i}\left[L^{S}-\beta \frac{1-\theta_{R}}{w}\right] ; \quad i=L, R
$$

Define conventional total factor productivity growth $\hat{Q}_{T F P}$ as the growth of output minus the appropriately weighted growth of inputs which, using (15), can be written as $\hat{Q}_{T F P} \equiv \hat{Y}-\left[\theta_{R} \hat{R}+\left(1-\theta_{R}\right) \hat{L}\right]=\theta_{R} \hat{Q}_{R}+\left(1-\theta_{R}\right) \hat{Q}_{L}$, where the second equality comes from (13). Using (30), (32) and (34), in an interior solution total factor productivity growth can be written as

$$
\hat{Q}_{T F P}=D+\left(\frac{\beta(1-\beta)}{\kappa_{L} \kappa_{R}}\right) \frac{\left(\theta_{R}-\kappa_{R}\right)^{2}}{w} .
$$

The following lemma shows that, in an interior solution, the dynamics of the whole model can be represented by a two-dimensional system in variables $\theta_{R}$ and $w$.

\section{Lemma 4 (System dynamics).}

In an interior solution with research in both sectors, the dynamics of the model evolve according to the following equations: 


$$
\begin{aligned}
& \hat{w}=\left(\rho+L^{S}\right)-\frac{\beta}{w}\left[2-\beta-\theta_{R}\right] \\
& \hat{\theta}_{R}=\frac{-\beta(1-v)\left(1-\theta_{R}\right)}{w}\left[\left(1+\frac{1-\beta}{\kappa_{L} \kappa_{R}}\right) \theta_{R}-\left(2-\beta+\frac{1-\beta}{\kappa_{L}}\right)+\left(\frac{L^{S}+\hat{Q}_{\mu}}{\beta}\right) w\right] .
\end{aligned}
$$

For a constant, non-positive, rate of mining efficiency improvements, $\hat{Q}_{\mu} \leq 0$, equations (40) and (41) define a phase diagram in the $\left(\theta_{R}, w\right)$ plane with negative sloped lines along which $w$ and $\theta_{R}$, respectively, are constant, and a negatively sloped saddlepath that leads to the interior steady state. However, in the short run, we can have positive mining efficiency improvements. In fact, since $\hat{Q}_{\mu}$ is time-varying, this is a non-autonomous system. In the $\left(\theta_{R}, w\right)$ plane, a change in mining efficiency improvements implies a rotation of the $\dot{\theta}_{R}=0$ locus around a point on the horizontal axis. Figure 2 depicts the phase diagram in the $\left(\theta_{R}, w\right)$ plane. We have included two $\dot{\theta}_{R}=0$ loci, one for $\hat{Q}_{\mu}>0$, the other for $\hat{Q}_{\mu}=0$. The figure also shows the non-negativity constraints for $D, D_{L}$ and $D_{R}$, which can be derived from (37) and (38). The phase-diagram shows the equilibrium path for the case in which the rate of mining efficiency improvements monotonically falls from a value that exceeds the discount rate to the value zero. This case results in an energy share that, in line with stylized fact 2bis, decreases initially, but eventually increases. In the remainder of this section we generalize the conditions under which the energy share falls and we check the implications for the other key variables from the list of stylized facts.

We now show that it is possible to find initial values for the key variables such that the short term dynamics of the model is qualitatively consistent with all the stylized facts reported above. Since fact 7 refers to the elasticity of substitution, which is modeled as a parameter, we impose $v<1$ as an assumption for this parameter and investigate the possibility to generate facts 1-6 as (short-run) outcomes of the model.

\section{Lemma 5 (calibration: matching the model dynamics to the stylized facts)}

If the initial conditions and parameters are chosen to satisfy all of the following:

(i) $\theta_{R}(0)=\kappa_{R}$,

(ii) $\rho<\hat{Q}_{\mu}(0)<L^{S}(1-\beta) / \kappa_{L}$,

(iii) $\rho<\hat{Q}_{\mu}(t)$ for $t \in[0, T)$, 
then there is a value $T_{1}>0$ such that, if $T \geq T_{1}$, the model is consistent with stylized facts 1-7.

Condition (i) implies that the energy share that is observed in the data provides the value for the cost parameter $\kappa_{R}$. The model is calibrated to generate the desired (observed) initial value for the energy share by choosing the initial value of the resource stock. ${ }^{15}$ Condition (ii) implies that extraction efficiency improvements are initially lower and upper bounded, what is a sufficient condition for stylized fact 4 to hold, while (iii) ensures that the economy benefits long enough from efficiency improvements in energy supply so that the energy share can fall over time and energy supply grows.

We can now start from this "qualitative calibration" in Lemma 5 to explore what developments the model predicts.

\section{Proposition 3 (dynamics and steady state of the qualitatively calibrated model)}

After calibrating the short-run dynamics of the model (with endogenous factor-augmenting technological progress and exogenous improvements in mining efficiency) to stylized facts 16 through making assumptions (i)-(iii) from Lemma 5, we find that over time

(i) the energy share initially falls; its long-run value exceeds its initial value;

(ii) technological change starts neutral but becomes increasingly energy-using initially; in the long run it is energy-saving;

(iii) per capita income growth will stabilize at a lower long-run value as compared to the initial value. There will be positive growth in the long-run if and only if the following condition holds: $\kappa_{R} \hat{Q}_{\mu}(\infty)+L^{S} \geq \frac{\left(1-\beta \kappa_{R}\right) \rho}{1-\beta}$

(iv) the relative price of energy (in terms of labor costs) is initially decreasing and it is increasing in the long run.

Proposition 3 shows that, once we account for endogenous technical change, the trend described by stylized fact 2 must be reversed: eventually energy scarcity will cause its share to increase over time. As discussed above, recent observations suggest an increasing trend for the energy share. Thus, we find that calibrating the model for $t=0$ to facts $1-6$, with declining

\footnotetext{
${ }^{15}$ For any combination of initial energy share and path of mining efficiency, $\left\{\theta_{R}(0), \hat{Q}_{\mu}(t)\right\}$, the system in (39)(40) defines $w(0)$, such that $\theta_{R}(t)$ and $w(t)$ approach constants (their steady state values) in the long run. Along the associated path, cumulative extraction has to equal the initial stock $E(0)$ to satisfy the transversality condition in (21). Since $E(0)$ does not enter any of the other equations, we can choose $E(0)$ to accomplish this.
} 
energy share in particular, generates an increasing energy share later on, thus replicating stylized fact 2 bis. This reversal cannot be adequately explained with constant exogenous rates of technical change (cf. Proposition 2). Therefore, studying the direction of technical change turns out to be crucial to explain the observed trends in energy use and economic growth. Under endogenous directed technical change, the initial improvements in mining efficiency induce energy-using technical change. Nevertheless, in the long, when the possibilities to increase mining efficiency are exhausted, technical changes becomes necessarily energysaving, and a higher energy dependence (higher energy share) can no longer be avoided. As a matter of fact, we conclude that, in the long run, the bias of technical change converges to $\hat{Q}_{R}(\infty)-\hat{Q}_{L}(\infty)=\rho-\hat{Q}_{\mu}(\infty)>0$, which is the knife-edge condition displayed in Lemma 3 , part (iii) (for more details, see the proof of Proposition 3 in Appendix A). On the other hand, we also conclude that the higher long-run energy share comes with a lower long-run growth rate and the possibility to have a positive growth rate in the long run depends on extraction efficiency gains remaining strong enough in the long run as compared to the discount rate.

\subsection{Dynamics and turning points with non-accelerating mining efficiency improvements}

One desirable feature of both Lemma 5 and Proposition 3 is that they are general results in the sense that they allow to calibrate the model and characterize its dynamic evolution without any specification for the path of mining improvements. They only use the assumptions that initial improvements should be (upper and lower) bounded in the short run and that in the long run mining efficiency cannot keep improving, but do not use any additional assumption on the specific time evolution of $Q_{\mu}(t)$. As a consequence, Proposition 3 characterizes only what happens at time zero and compares this to what happens in the long run. It does not characterize what happens in the "medium run", since this will depend on how mining efficiency improvements proceed between time zero and the long run. We now explore the implications of an additional assumption, regarding the medium run mining efficiency improvements: if we assume these do never accelerate over time, we find the results as summarized in the following proposition.

\section{Proposition 4 (dynamics with non-accelerating mining efficiency improvements)}

After calibrating the short-run dynamics of the model (with endogenous factor-augmenting technological progress and exogenous improvements in mining efficiency) to stylized facts 1-

6 through making assumptions (i)-(iii) from Lemma 5, and assuming in addition that $\hat{Q}_{\mu}(t)$ is non-increasing over time, we find that over time 
(i) the energy share first falls then rises to a higher than initial level;

(ii) total research effort $(D)$ first falls, then rises;

(iii) technological change becomes more and more energy-using first, but then gradually becomes less energy-using and turns into energy-saving;

(iv) total factor productivity growth either initially declines and then rises or it initially declines, then rises, then declines again and finally rises.

The dynamics of the (qualitatively calibrated) model imply that the energy share continues decreasing for a while as long as mining efficiency improvements are large enough. At this stage, energy is abundant and there are little incentives for energy-saving technological change. However, when these improvements become smaller and smaller, and scarcity of resources increases, the energy share starts increasing. This triggers energy-saving technological change, which slows down the increase in the energy share. Indeed, in the long run, the model predicts a constant energy share, as a result of the interaction between resource extraction and endogenous directed technological change. These results allow us to be more precise about the economic forces driving the energy share, which cannot be done if technical change is not modeled. (cf. Proposition 2).

Research effort increases after oil has peaked. This is because with scarcer energy, labor productivity in goods production falls, the wage falls, and R\&D becomes relatively cheaper. However, per capita income growth is lower in the steady state than at time zero: the increase in total factor productivity is not enough to offset the decline in per capita energy use and the decline in mining efficiency improvements.

Under the calibration assumptions used in Proposition 4, we can get some additional insights about the timing of the turning points for the relevant variables although, at the current level of generality of the model, it is not possible to determine with certainty the timing of all of them. Define $t_{X}$ as the time at which variable $X$ changes its trend. For example, $t_{R}$ is the turning point for energy, (characterized by $\hat{R}\left(t_{R}\right)=0$ ) and so on. From equation (33) it is obvious that $t_{\theta_{R}}<t_{Q_{R} / Q_{L}}$, i.e., the turning point for $\theta_{R}$ happens before the bias of technical change ceases to decrease and starts to increase. The reason is that the minimum of the technical bias is reached when $\theta_{R}=\kappa_{R}$ what, apart from the initial point, only happens when $\theta_{R}$ is increasing. From the phase diagram it is clear that $t_{w}<t_{\theta_{R}}$, i.e., $w$ reaches a maximum before $\theta_{R}$ reaches a minimum. Using this results in combination with equation (36), we 
conclude that for any time between $t_{w}$ and $t_{\theta_{R}} L$ is increasing (and, hence, $D$ is decreasing), what implies that $t_{w}<t_{\theta_{R}}<t_{L}=t_{D}$ i.e., both $L$ and $D$ reach their respective turning points after $\theta_{R}$ does. Recalling that the energy share can be taken as a measure (or at least a sign) of resource scarcity, we have the following interpretation of these results: scarcity is a fundamental driving force for the technological bias and the distribution of the labor force between production and research but these variables do not get reversed just because resource scarcity starts to increase, but only when such scarcity is strong enough.

Other comparisons between the turning points of key variables are not so straight forward and, actually, they turn out to depend on the intensity of mining efficiency improvements. Regarding the timing of $t_{R}$ and $t_{\theta_{R}}$, using equation (27) and the constant

population assumption we know $\hat{\theta}_{R}\left(t_{R}\right)=\rho-\hat{Q}_{\mu}\left(t_{R}\right)$ and we can find two cases. If at $t_{R}$, mining improvements are strong enough (specifically, if $\hat{Q}_{\mu}\left(t_{R}\right)>\rho$ ) then we have $\hat{\theta}_{R}\left(t_{R}\right)<0$ and we conclude $t_{R}<t_{\theta_{R}}$, i.e., the turning point for $R$ happens before that of $\theta_{R}$. If, on the contrary, at the same point in time these improvements do not exist or are not strong enough (i.e., $\hat{Q}_{\mu}\left(t_{R}\right)<\rho$ ) we have $t_{R}<t_{\theta_{R}}$ and so energy use starts decreasing before the energy share turns up. In the latter case we know for sure that $t_{R}<t_{\theta_{R}}<t_{Q_{R} / Q_{L}}$. The interpretation for his fact is that mining improvements causes $R$ to start decreasing, as a symptom of physical scarcity, before we observe an increase in economic scarcity as measured by the energy share.

\subsection{Numerical illustration}

Although the aim of our study is to provide a qualitative understanding of the driving forces behind the empirical facts on energy use rather than a numerical calibration, it is also useful to present here a numerical exercise to illustrate how the model can be calibrated to mimic to some extent the observed trends.

To calibrate the model, we pick a conventional value for $\rho$ and choose $\kappa_{R}$ to be equal to the energy share in the 1960s. We choose both $\sigma$ and $v$ in line with the empirical evidence on substitution elasticities. In the estimates by Van der Werf (2008), the elasticities are in the interval $(0.17,0.61)$. This restricts our choice of $\beta$, which should be large enough to make the elasticity $v$ not too big (notice that $v$ is the weighted average of the elasticity of substitution between the primary factor and intermediates within the sectors, which equals 1 , and the 
elasticity of substitution between the sectors, $\sigma$ ). We choose $\beta=0.75$ and $\sigma=0.2$, which gives $v=0.4$. We then choose $L^{S}=0.14$ to generate a steady state growth rate that is $1.2 \%$.

The only parameter left to choose is $\hat{Q}_{\mu}(t)$. We choose a path for this variable such that the path of $\theta_{R}$ is smooth with one turning point and follows the path that is observed in the data (Figure 3). We concentrate on the long-run pattern that illustrates the trend from the perspective of the early post-war period ignoring the peak in the energy share between 1972 and 1985, to acknowledge that the events in this period implied shocks that are not in our model and could not have been anticipated by markets in the 1950s. This calibration illustrates the trend that markets would anticipate before the oil crisis. Figure 3 displays the observed data on the energy share together with the smoothed version we have constructed by disregarding the oil crises. Then, we find the path for energy mining improvements, $\hat{Q}_{\mu}(t)$, that is required to generate this smoothed trend for $\theta_{R}$ and we use this data to simulate the model. The constructed series of $\hat{Q}_{\mu}(t)$ is displayed in Figure 4. Note that this series is rather consistent with the empirical evidence reviewed in section 3, with mining efficiency clearly improving during the first post-war decades and those improvements disappearing and getting reversed by the end of the century. Based on this close correlation we expect that a model with endogenous extraction costs can easily replicate the stylized facts.

Figure 5 displays the normalized observed data $(1953=1)$ on per capita energy consumption, the energy share and the relative price of energy. Figure 6 show the counterpart of the same series generated by the calibrated version of our model. By construction the calibrated model trace the energy share closely. However, we see that endogenously it also traces the behavior of energy use and energy prices quite closely. In our calibration, $\mathrm{Q}_{\mathrm{L}}$ and $\mathrm{Q}_{\mathrm{R}}$ grow at rates between $0.9 \%$ and $2.4 \%$ which is in line with the empirical evidence.

\section{Discussion}

The model outlined above is deliberately simple. Various important issues could be studied in an extended versions of the model. In this section we briefly discuss two of these issues, leaving full-fledged modeling to future work. 


\subsection{Endogenous extraction costs}

Above we concluded that the model can be successfully calibrated if technical change in the mining sector makes extraction costs decline over time. We have not modeled how changes in extraction costs could be the results of various forces. A version of the model with endogenous innovation has too many state variables to allow for an analytical solution of the transition dynamics. However, also with endogenous extraction costs, a pattern of declining improvements over time can be expected to be the outcome. Incentives for investment in extraction cost reductions should come from a sufficient market size for cheaper drilling techniques and from low enough costs to develop these techniques. The maximum market size is given by total expenditures on extraction costs, $\mu R w_{R}$, while the cost of R\&D could be - in line with the assumption on the cost of R\&D in the production sector - declining in the stock of knowledge and increasing in the wage of researchers, say $w_{D} / Q_{\mu}$. The benefit/cost ratio is therefore proportional to:

$$
\frac{\mu R w_{R}}{w_{D} / Q_{\mu}}=\frac{\mu}{1+\mu}\left(\frac{\theta_{R}}{w}\right),
$$

where the equality follows after substituting the definitions of $Q_{\mu}=1 /(1+\mu)$, $\theta_{R}=w_{R} R / p_{Y} Y$, and $w=w_{D} / p_{Y} Y$. Since this benefit/cost ratio declines with reductions in extraction costs (for given $\theta_{R} / w$ ), we can expect that it falls over time if there would be ongoing technical change in extraction - a situation which would lead to the endogenous slowing down of R\&D in extraction. To see this, consider the Phase diagram from the previous section with a constant $\hat{Q}_{\mu}(t)$ : since the isoclines don't shift, the variables $\theta_{R}$ and $w$ then gradually approach their constant values, while $\mu$ keeps falling.

The basic reason why $R \& D$ in mining must stop is that we put a limit on efficiency, i.e. $\mu$ cannot be negative. The alternative assumption would be that - as a result of very powerful technical change - the term $(1+\mu)$ can go to zero, meaning that when we arrive at this situation, the use resource in production $(R)$ does not reduce the stock of resources, $\mathrm{E}$, for future use. Resource scarcity would then be eliminated: we would leave the Hotelling world and enter a cornucopian world that is beyond the scope of this paper. 


\subsection{Policy implications}

What are the policy implications of our findings? We studied the market equilibrium with the two usual market imperfections: researchers ignore the knowledge spillovers on other researchers and intermediate goods producers set monopoly prices. Optimal policy would require correction through subsidies to intermediate goods production and R\&D. An important consequence of the knowledge spillovers is that the market invests too little in energy efficiency improvements in the long run. We formulate this in the following proposition:

\section{Proposition 5 (Optimal energy policy).}

In the long-run market equilibrium, the energy share is too high as compared to the social optimum.

To compensate for the fall in resource inputs in the long run, the market generates energybiased technical change. In other words, in the long-run the demand for energy-related knowledge grows faster than the demand for labor-related knowledge. The value of spillovers in energy-related research is accordingly larger. However, researchers are not rewarded for the creation of knowledge spillovers so that research is not strongly enough directed to energy efficiency improvements. As a result, the laissez-faire market economy is too much dependent on energy.

Energy dependence is a concern in the real world for two reasons that are not in the model. First, individual countries may need to import energy from insecure sources. To avoid the social costs of potential supply disruptions, these countries could have an incentive to invest additionally in energy-saving. Second, energy use causes emissions, greenhouse gas and local pollutants in particular. Both externalities could be handled by a tax on energy use. We expect that in the setting of our model, the effect of such a tax would be similar to a change in mining costs, since it changes the cost for resource owners. In particular, both in the context of climate change policy and energy security policy, the aim of the policy would be let cumulative emissions (or energy imports) grow slower over time, as compared to the laissez-faire economy and this would require an energy tax that is declining over time (see Sinclair 1992), similar to a situation in which extraction costs fall over time. The model ignores pollution externalities. If resource uses causes pollution emissions a pollution tax should be levied on the energy emissions fossil fuels 


\section{Conclusions}

We have developed an endogenous growth model that connects the rate and direction of technical change to energy use and optimal resource extraction within a dynamic general equilibrium framework. The novel element is that both technical change and resource use are assumed to be endogenous variables resulting from market arbitrage activities by rational optimizing agents.

This model is shown to be compatible with the key stylized facts about economic growth and energy use observed during the second half of the $20^{\text {th }}$ century. Specifically, we show that when resource extraction costs steadily decline, the model generates steadily increasing per capita energy use and decreasing energy share in GDP.

We also conclude that if we take the dynamics of the model towards its steady state as an indication for possible future trends, some important postwar trends in energy use and prices can be expected to be reversed in the future. Indeed, aggregate energy-related data on the first decade of the $21^{\text {st }}$ century could be interpreted as confirming our prediction, although these recent data could also reflect temporary shocks rather than a change of the long-run trend. First, the model predicts that per capita energy use will start declining at some point in the future, and will keep declining in the long run. Since by definition the stock of nonrenewable resources is finite, increasing energy use is unsustainable. The increased scarcity of resources shifts technical change progressively towards energy-saving technological change. This stimulates total factor productivity growth, which thus partly offsets the negative impact of oil having peaked.

Second, the effect of efficiency gains in resource-extraction technologies is likely to become progressively smaller as compared to the exhaustion effect, which means that the observed decrease in energy prices relative to labor prices is likely to be reversed in the long run as the energy price evolution converges to the standard Hotelling rule. In a similar way, the evolution of energy share is also likely to show a U-shaped pattern. Some of this reversal of the decline in energy dependency appeared in recent years. Our model therefore suggests that the spikes in the energy share around the oil crises and the high current level might be interpreted as the start of a structurally higher energy dependency.

Our model highlights what may drive the dynamics of growth and energy scarcity over time. The exhaustion of essential natural resources, in itself, does not necessarily lead to lower growth in future. Rather, it is the exhaustion of the potential to improve the efficiency with which we exploit these physical resources that might slow down growth. 


\section{References}

Acemoglu, D. (1998) "Why do new technologies complement skills? Directed technical change and wage inequality," Quarterly Journal of Economics 113, 1055-1089.

Acemoglu, D. (2002) "Directed Technical Change", Review of Economic Studies 69, 781-809.

Acemoglu, D., P. Aghion, L. Bursztyn and D. Hemous (2012) "The Environment and Directed Technical Change" American Economic Review 102, 131-166.

Barbier, E.B. (1999) "Endogenous Growth and Natural Resource Scarcity", Environmental and Resource Economics 14, 51-74.

Barnett, H. (1979) Scarcity and Growth Revisited. In Scarcity and Growth Reconsidered, edited by V. K. Smith. Baltimore: Johns Hopkins University Press for Resources for the Future, $163-217$.

Barnett, H., and C. Morse (1963) Scarcity and Growth: The Economics of Natural Resource Availability. Baltimore: Johns Hopkins University Press for Resources for the Future.Benchecroun, H. and C. Withagen (2011), "The optimal depletion of exhaustible resources: a complete characterization", Resource and Energy Economics $33,612-636$.

Benchekroun, H. and C. Withagen (2011) "The optimal depletion of exhaustible resources: A complete characterization", Resource and Energy Economics 33, 612-636.

Berck, P. and M. Roberts (1996) "Natural Resource Prices: Will they ever turn up?" Journal of Environmental Economics and Management 31, 65-78.

Caselli, F. (2005) "Accounting for Cross-country Income Differences" in Handbook of Economic Growth, Volume 1A. Edited by Philippe Aghion and Steven N. Durlauf. Amsterdam: Elsevier.

Chakravorty, U., M. Moreaux, M. Tidball (2008) "Ordering the Extraction of Polluting Nonrenewable Resources", American Economic Review 98, 1128-1144.

Chermak, J. and R.H. Patrick (1995) "A Well-Based Cost Function and the Economics of Exhaustible Resources: The Case of Natural Gas", Journal of Environmental Economics and Management 28, 174-189.

Dasgupta, P.S. and G.E. Heal (1974) "The Optimal Depletion of Exhaustible Resources". Review of Economic Studies (Symposium), 3-28.

Dasgupta, P.S. and G.E. Heal (1979) The Economics of Exhaustible Resources, Cambridge: Cambridge University Press.

Deffeyes, K.S. (2001) Hubbert's Peak: The Impending World Oil Shortage. Princeton: Princeton University Press.

Di Maria, C. and S. Valente (2008) "Hicks meets Hotelling: the direction of technical change in capital-resource economies", Environment and Development Economics 13, 691717.

Dixit A.K. and J.E. Stiglitz (1977) "Monopolistic Competition and Optimum Product Diversity", American Economic Review 67, 297-308.

EIA (Energy Information Administration) (2011) "Performance Profiles of Major Energy Producers 2009" EIA, DOE/EIA-0206(09), Washington DC.

EIA (2012) "Annual Energy Review", US Energy Information Administration. http://www.eia.gov/totalenergy/data/annual/index.cfm, Last accessed September 25, 2012.

Fagan, M.N. (1997) "Resource Depletion and Technical Change: Effects on U.S. Crude Oil Finding Costs from 1977 to 1994", The Energy Journal 18, 91-106.

Gaudet, Gérard (2007). "Natural Resource Economics under the Rule of Hotelling," Canadian Journal of Economics 40, 1033-1059. 
Grimaud, A. and L. Rougé (2003) "Non Renewable Resources and Growth with Vertical Innovations: Optimum, Equilibrium and Economic Policies“, Journal of Environmental Economics and Management 45, 433-453.

Grossman, G.M. and E. Helpman (1991) Innovation and Growth in the Global Economy. The MIT Press, Cambridge, M.A.

Groth, C. and P. Schou (2002) "Can non-renewable resources alleviate the knife-edge character of endogenous growth?", Oxford Economic Papers 54, 386-411.

Groth, C. and P. Schou (2005) "Growth and Non-renewable Resources: Does Capital Taxation Matter?" mimeo, Copenhagen University.

Hall, D.C. and J.V. Hall (1984) "Concepts and Measures of Natural Resource Scarcity with a Summary of Recent Trends", Journal of Environmental Economics and Management $11,363-379$.

Heal, G. (1976) "The Relationship Between Price and Extraction Cost for a Resource with a Backstop Technology", Bell Journal of Economics 7, 371-378.

Hotelling, H. (1931) "The Economics of Exhaustible Resources", Journal of Political Economy 39, 137-175.

Howitt, P. (1999) "Steady Endogenous Growth with Population and R\&D Inputs Growing" Journal of Political Economy 107, 715-730.

Johnson, M, F. Bell, and I. Bennett (1980) "Natural resource scarcity: Empirical evidence and public policy", Journal of Environmental Economics and Management 7,256-271.

Jones, C.I. (2002) Introduction to Economic Growth, Second Edition, New York: W.W. Norton.

Jorgenson, D.W. (1984) “The Role of Energy in Productivity Growth” in J.W. Kendrick (ed.) International Comparisons of Productivity and Causes of the Slowdown, Cambridge MA: Ballinger, 279-323.

Jorgenson, D.W. and B.M. Fraumendi (1981) "Relative prices and Technical Change" in E. Berndt and B. Field (eds.) Modeling and Measuring Natural Resource Substitution, Cambridge: MIT Press.

Krautkraemer, J.A. (1998) "Nonrenewable Resource Scarcity" Journal of Economic Literature 36, 2065-2107.

Krautkraemer, J.A. (2005) "Economics of Scarcity: The State of the Debate" in R.D. Simpson, M.A. Toman and R. U. Ayres (eds.), Scarcity and Growth revisited, Washington DC: Resources for the Future Press.

Kuper and Van Soest (2002) "Path dependency and input substitution: implication for energy policy modeling" Energy Economics 25, 397-407.

Neumayer, E. (2003). Weak versus Strong Sustainability; exploring the limits if two opposing paradigms. Cheltenham: Edward Elgar.

Nordhaus, W. (1994) Managing the Global Commons: The Economics of Climate Change, Cambridge, MA: MIT Press.

Popp, D. (2002) "Induced Innovation and Energy Prices", American Economic Review 92, $160-180$.

Prywes, M. (1986) "A nested CES approach to capital-energy substitution", Energy Economics 8, 22-28.

Romer, P.M. (1990) "Endogenous Technological Change”, Journal of Political Economy 98, S71-103.

Samuelson, P.A. (1954) "The transfer problem and transport costs II: Analysis of the effects of trade impediments", Economic Journal 64, 147-162.

Sinclair, P. (1992) "High Does Nothing and Rising is Worse: Carbon Taxes Should Keep Declining to Cut Harmful Emissions", The Manchester School 60, 41-52. 
Smulders, S. and M. de Nooij (2003) "The Impact of Energy Conservation on Technology and Economic Growth", Resource and Energy Economics 25, 59-79.

Sue Wing, I. (2008). "Explaining the Declining Energy Intensity of the U.S. Economy", Resource and Energy Economics 30, 21-49.

Sue Wing, I. and R.S. Eckaus (2007). The Decline in U.S. Energy Intensity: Its Origins and Implications for Long-Run CO2 Emission Projections, Energy Policy 35: 5267-5286.

Tahvonen, O. and S. Salo (2001) "Economic Growth and Transition Between Renewable and Nonrenewable Energy Resources" European Economic Review 45, 1379-1398.

Van der Ploeg, F. and C. Withagen (2011) "Too much coal, too little oil." Journal of Public Economics 96, 72-77.

Van der Werf, E. (2008) "Production functions for climate policy modeling: an empirical analysis", Energy Economics 30, 2964-2979. 


\section{Appendix A: Proofs.}

\section{Proof of Lemma 1}

Using (17), (20) and differentiating (4) we get $\hat{w}_{R}-\hat{w}_{L}=\rho-\hat{Q}_{\mu}+\hat{L}-\hat{L}^{S}-\hat{\theta}_{L}$. We differentiate (11) and (12), and use the result to eliminate $\hat{w}_{R}-\hat{w}_{L}$ and $\hat{\theta}_{L}$, respectively. After rearranging we obtain (22). Differentiating (12) and using (22) we get (23). Differentiating (4), (5) and (12), we get (24). Finally, (25) and (26) follow from (15) and (22).

QED.

\section{Proof of Lemma 2}

From (22), (23) and (24), we get

$$
\begin{gathered}
\hat{R}-\hat{L}^{s}>0 \Leftrightarrow \hat{Q}_{R}-\hat{Q}_{L}<\hat{L}-\hat{L}^{s}+\frac{n\left(\hat{Q}_{\mu}-\rho\right)}{1-n}, \\
\hat{\theta}_{R}<0 \Leftrightarrow \hat{Q}_{R}-\hat{Q}_{L}>\left(\hat{L}-\hat{L}^{s}\right)-\left(\hat{Q}_{\mu}-\rho\right), \\
\hat{w}_{R}-\hat{w}_{L}<0 \Leftrightarrow\left(\hat{Q}_{R}-\hat{Q}_{L}\right)>\frac{\left(1-n \theta_{R}\right)}{\theta_{R}(1-n)}\left[\left(\hat{L}-\hat{L}^{s}\right)-\left(\hat{Q}_{\mu}-\rho\right)\right],
\end{gathered}
$$

which, using the definition of $n$, can be combined to get the bounds displayed in the lemma.

QED.

\section{Proof of Proposition 1}

Using $\hat{Q}_{R}=\hat{Q}_{L}$ in (25), it follows that condition (ii) is both necessary and sufficient for fact 4 . Using again $\hat{Q}_{R}=\hat{Q}_{L}$ and manipulating equations (22), (23) and (24) we find

$$
\begin{array}{ccc}
\hat{R}-\hat{L}^{s}>0 & \Leftrightarrow & \hat{Q}_{\mu}>\rho-\frac{(1-n)}{n} \cdot\left(\hat{L}-\hat{L}^{s}\right), \\
\hat{\theta}_{R}<0 & \Leftrightarrow & \hat{Q}_{\mu}>\rho+\hat{L}-\hat{L}^{s}, \\
\hat{w}_{R}-\hat{w}_{L}<0 & \Leftrightarrow & \hat{Q}_{\mu}>\rho+\hat{L}-\hat{L}^{s} .
\end{array}
$$

Combining these expressions, we conclude that (i) is necessary and, moreover, it is sufficient for facts 1, 2 and 3. Finally, facts 1 and 4 together imply the fulfillment of fact 5 . Therefore, conditions (i) and (ii) are also sufficient for fact 5.

QED. 


\section{Proof of Lemma 3}

From (23), we have that $\hat{Q}_{\mu}+\hat{Q}_{R}-\hat{Q}_{L}>\rho$ implies $\hat{\theta}_{R}<0$ and, therefore, $\lim _{t \rightarrow \infty} \theta_{R}(t)=0$. If, on other hand, $\hat{Q}_{\mu}+\hat{Q}_{R}-\hat{Q}_{L}<\rho$ then $\hat{\theta}_{R}>0$ and $\lim _{t \rightarrow \infty} \theta_{R}(t)=1$. Expressions for $\hat{Y}-\hat{L}^{S}$ follow from (26).

QED.

\section{Proof of Proposition 2}

Define $\hat{Q}_{\rho} \equiv \hat{Q}_{\mu}+\hat{Q}_{R}-\hat{Q}_{L}-\rho$. From (23) it follows that, if $\hat{Q}_{\rho}>0, \lim _{t \rightarrow \infty} \theta_{R}(t)=0$ and, therefore, $\lim _{t \rightarrow \infty} n(t)=v$. On the other hand, if $\hat{Q}_{\rho}<0, \lim _{t \rightarrow \infty} \theta_{R}(t)=0$ and, therefore, $\lim _{t \rightarrow \infty} n(t)=1$. Using these results in (22)-(26) and checking the three relevant cases $\left(\hat{Q}_{\rho} \leq 0\right)$, we get the following long-run expressions, from which results (i)-(iv) are immediate:

$$
\begin{aligned}
& \hat{R}-\hat{L}=-\left(\rho-\hat{Q}_{\mu}\right)-\max \left\{0,(1-v) \hat{Q}_{\rho}\right\}, \\
& \hat{\theta}_{R}=\min \left\{0,-(1-v) \hat{Q}_{\rho}\right\}, \\
& \hat{w}_{R}-\hat{w}_{L}=\rho-\hat{Q}_{\mu}, \\
& \hat{Y}-\hat{R}=\hat{Q}_{R}-\max \left\{0, v \hat{Q}_{\rho}\right\}, \\
& \hat{Y}-\hat{L}=\min \left\{\hat{Q}_{\mu}+\hat{Q}_{R}-\rho, \hat{Q}_{L}\right\} .
\end{aligned}
$$

QED.

\section{Proof of Lemma 4}

(40) immediately follows after using (37) in (35) to eliminate $D$. From (36) we get $\hat{L}=-\hat{\theta}_{R} \theta_{R} /\left(1-\theta_{R}\right)-\hat{w}$. Using this expression to eliminate $\hat{L},(33)$ to eliminate the growth of technological bias and (40) to eliminate $\hat{w},(41)$ is obtained.

QED.

\section{Proof of Lemma 5}

It follows from (33) that (i) guarantees the fulfillment of stylized fact 6, $\hat{Q}_{R}(0)=\hat{Q}_{L}(0)$.

Differentiating (36), using (23) to eliminate $\hat{\theta}_{R}$, the definition for $n$, as well as (33) and (i), we get $\hat{L}(0)=(1-v) \kappa_{R}\left[\hat{Q}_{\mu}(0)-\rho\right]-\left(\kappa_{L}+v \kappa_{R}\right) \hat{w}(0)$ which, using (40), we can rewrite as

$$
\hat{L}(0)=(1-v) \kappa_{R}\left[\hat{Q}_{\mu}(0)-\rho\right]-\left(\kappa_{L}+v \kappa_{R}\right)\left[\rho+L^{S}-\frac{\beta\left(\kappa_{L}+1-\beta\right)}{w(0)}\right] .
$$


We define $\underline{w}$ and $\bar{w}$ as the values of $w$ associated to the intersection between the $\dot{\theta}_{R}=0$ locus and the $\dot{w}=0$ locus for the lowest and highest value of $\hat{Q}_{\mu}(t)$, respectively. We also define $w^{*} \equiv \beta\left(1-\beta+\kappa_{L}\right) /\left[L^{S}+\hat{Q}_{\mu}(0)\right]$ as the value of $w$ associated to the intersection between the $\theta_{R}=\kappa_{R}$ line and the $\dot{\theta}_{R}=0$ locus for $\hat{Q}_{\mu}(0)$.

By construction, in a steady state with constant $\theta_{R}$ and $w$, we have $\bar{w} \geq w \geq \underline{w}$. Moreover, the equilibrium dynamics imply

$$
\underline{w} \leq w(t) \leq \bar{w} \quad \text { for all } t \geq 0,
$$

since, otherwise, (40) implies that the solution would not converge to the steady state.

To get $\dot{\theta}_{R}(0)<0$ (stylized fact 2) $w(0)$ has to start above the $\dot{\theta}_{R}=0$ locus, that is $w(0)>w^{*}$. We now prove by contradiction that condition (ii) and a large enough $T$ guarantee this inequality. Suppose $w(0)<w^{*}$. Then, as long as $\rho<\hat{Q}_{\mu}$, it follows from (40) and (41) that $w$ would fall and $\theta_{R}$ increase over time. It also follows that if $\rho<\hat{Q}_{\mu}$ holds for a long enough period, $w$ would keep declining until it is smaller than $\underline{w}$, while $\theta_{R}$ is still below the steady state value. But according to (A.2), $w<\underline{w}$ cannot be an equilibrium. It follows that only $w(0)>w^{*}$ can be an equilibrium, which implies $\dot{\theta}_{R}(0)<0$. From (A.2), $w(0)>w^{*}$ is only feasible if $w^{*}<\bar{w}$, which requires $\hat{Q}_{\mu}(0)>\rho+\kappa_{L} \kappa_{R}(1-\beta)^{-1}\left[\rho-\max \left\{\hat{Q}_{\mu}(t)\right\}\right]$, which holds under condition (iii). This proves that stylized fact 2 is replicated with (iii) and sufficiently large $T .{ }^{16}$

Substituting (A.1) and (i) (this lemma) into (22), we see that (iii) and $w(0)<\bar{w}$, which was proven above, are sufficient to replicate stylized fact $\mathbf{1}$.

Substituting (A.1), (23) and (i) (this lemma) into (24), we see that stylized fact $\mathbf{3}$ is replicated for $w(0)>w^{*}$ (which was proven above). Hence if stylized fact 2 is replicated and (i) holds, stylized fact 3 is replicated as well.

It follows from (25), (i) (this lemma), (40), and $w(0)<\bar{w}$ that (ii) is sufficient to replicate stylized fact 4.

\footnotetext{
${ }^{16}$ Similarly, for given $T$, the length of period during which mining efficiency improves at a rate faster than $\rho$, we can ensure $\dot{\theta}_{R}(0)<0$ by increasing the rate of mining efficiency improvements before $T$.
} 
Since $\hat{Y}-\hat{L}_{p}=(\hat{Y}-\hat{R})+\left(\hat{R}-\hat{L}_{p}\right)$ and both terms in parentheses are positive if stylized facts 1 and 2 are satisfied, stylized fact 5 needs no further conditions.

QED.

\section{Proof of Proposition 3}

Solving (40) and (41) for the long-run energy share and wage rate from Lemma 3, we find:

$$
\begin{aligned}
& \theta_{R}(\infty)=\kappa_{R}+\left(\kappa_{L}+1-\beta\right) a /(a+b)>\kappa_{R}=\theta_{R}(0), \\
& w(\infty)=\beta(1-\beta)\left(\kappa_{L}+1-\beta\right) /(a+b),
\end{aligned}
$$

where $a \equiv \kappa_{L} \kappa_{R}\left[\rho-\hat{Q}_{\mu}(\infty)\right]$ and $b \equiv\left(L^{S}+\rho\right)(1-\beta)$. The calibration in Lemma 5 ensures $\theta_{R}(0)=\kappa_{R}<\theta_{R}(\infty)$ and $\dot{\theta}_{R}(0)>0$. This proves part (i).

Technological change is energy-using (energy-saving) if $\hat{Q}_{R}-\hat{Q}_{L}<(>) 0$. The short run part of (ii) follows from equation (33) and the initial condition of $\theta_{R}$. Concerning the long run result, using the above expressions for $\theta_{R}(\infty)$ and $w(\infty)$ in (33), we get $\hat{Q}_{R}(\infty)-\hat{Q}_{L}(\infty)=\rho-\hat{Q}_{\mu}(\infty)>0$.

Substituting (31), (33), and (38) into (26), we express per capita growth as a function of $w, \theta_{R}$ and $\hat{L}$. To find long-run per capita growth from the resulting expression, we substitute $\hat{L}=0$ and the long-run values of $w$ and $\theta_{R}$, which yields:

$$
\hat{Y}(\infty)=\hat{Q}_{L}(\infty)=\frac{(1-\beta) L^{S}-\kappa_{L} \rho-(1-\beta) \kappa_{R}\left[\rho-\hat{Q}_{\mu}(\infty)\right]}{1-\beta+\kappa_{L}} .
$$

Noting that the denominator of this fraction is positive and using the assumption $\kappa_{L}+\kappa_{R}=1$, we obtain the following condition for the long-run growth to be positive:

$$
\hat{Y}(\infty)=\hat{Q}_{L}(\infty) \geq 0 \Leftrightarrow \kappa_{R} \hat{Q}_{\mu}(\infty)+L^{S} \geq \frac{\left(1-\beta \kappa_{R}\right) \rho}{1-\beta}
$$

Short-run growth can be expressed, using (A.1) and $\theta_{R}(0)=\kappa_{R}$, as:

$$
\hat{Y}(0)=\kappa_{R}\left[L^{S}+\hat{Q}_{\mu}(0)\right]-\rho+\left(\kappa_{L}-\beta\right) \kappa_{L} \beta / w(0) .
$$

If $\kappa_{L}=\beta$, we can compare long-run and short-run growth without knowing $w(0)$ :

$$
\hat{Y}(0)-\hat{Y}(\infty)=(1-\beta)\left[\hat{Q}_{\mu}(0)-\rho\right]+(1-\beta) \kappa_{R}\left[\rho-\hat{Q}_{\mu}(\infty)\right]>0 .
$$

If $\kappa_{L}<\beta$, we find a lower bound for short-run growth, using $w(0)>w^{*}$ :

$$
\hat{Y}(0)>\left(\frac{1-\beta}{1-\beta+\kappa_{L}}\right) L^{S}-\left(\frac{\kappa_{L}}{1-\beta+\kappa_{L}}\right) \rho+\left(\frac{1-\beta}{1-\beta+\kappa_{L}}\right)\left[\hat{Q}_{\mu}(0)-\rho\right] .
$$


If $\kappa_{L}>\beta$, we find a lower bound for short-run growth, using $w(0)<\bar{w}$ and assuming $\hat{Q}_{\mu}(0)=\max _{t \geq 0} \hat{Q}_{\mu}(t)$ :

$$
\hat{Y}(0)>\left(\frac{1-\beta}{1-\beta+\kappa_{L}}\right) L^{S}-\left(\frac{\kappa_{L}}{1-\beta+\kappa_{L}}\right) \rho+\left(\frac{1-\beta}{1-\beta+\kappa_{L}}+\frac{\kappa_{L} \kappa_{R}}{1-\beta}\right) \kappa_{R}\left[\hat{Q}_{\mu}(0)-\rho\right] .
$$

Since $\rho-\hat{Q}_{\mu}(0)<0<\rho-\hat{Q}_{\mu}(\infty)$, we find $\hat{Y}(\infty)<\hat{Y}(0)$ for all three cases. This proves part (iii).

QED.

\section{Proof of Proposition 4}

To prove part (i), define $\theta_{R}^{*}(t)$ as the value of $\theta_{R}$ associated to the intersection between the $\dot{\theta}_{R}=0$ and the $\dot{w}=0$ locus. Since the system is non-autonomous, $\theta_{R}^{*}(t)$ is time-varying. Actually, under the assumption that $\hat{Q}_{\mu}(t)$ is non-increasing over time, $\theta_{R}^{*}(t)$ monotonically increases. Then, as illustrated in the phase-diagram, the dynamics of the system can be divided in two stages: In the first stage, $\theta_{R}>\theta_{R}^{*}(t)$ and saddle-path stability implies that $\theta_{R}$ is decreasing. In the second stage $\theta_{R}(t) \leq \theta_{R}^{*}(t)$ and saddle-path stability implies that $\theta_{R}(t)$ is increasing. The fact that $\theta_{R}(0)<\theta_{R}(\infty)$ ensures that stage 2 will be reached.

To derive (ii), we plot the iso- $D$ lines in the $\left(\theta_{R}, w\right)$ phase diagram: from (37), these are given by $w=\left(1-\theta_{R}\right) \beta /\left(L^{S}-D\right)$ and their slopes are negative and steeper than the $\dot{w}=0$ locus and a fortiori steeper than the equilibrium path. Combining these isoclines with the equilibrium $\left(\theta_{R}, w\right)$ trajectory, we find (ii).

To derive (iii), we plot the iso- $\left(\hat{Q}_{R}-\hat{Q}_{L}\right)$ lines in the $\left(\theta_{R}, w\right)$ phase diagram: from (33), these are rays emanating from point $\kappa_{R}$ on the horizontal axis defined by $w=\beta(1-\beta)\left(\kappa_{L} \kappa_{R}\right)^{-1}\left(\hat{Q}_{R}-\hat{Q}_{L}\right)^{-1}\left(\theta_{R}-\kappa_{R}\right)$. Combining these isoclines with the equilibrium $\left(\theta_{R}, w\right)$ trajectory, we find (iii).

Substituting (37) in (39), we find $\hat{Q}_{T F P}=L^{S}+(\beta / w)\left[\left(\theta_{R}-\kappa_{R}\right)^{2}(1-\beta) / \kappa_{R} \kappa_{L}+\theta_{R}-1\right]$, from which we derive iso-TFP-growth curves. These curves have a minimum at $\theta_{R}=\theta_{T} \equiv\left[1-\kappa_{L} / 2(1-\beta)\right] \kappa_{R}<\kappa_{R}$. From Lemma 5 we have $\theta_{R}(0)=\kappa_{R}$ and from part (i) of this proposition we have that $\theta_{R}$ first declines and then rises. We first infer from (40)-(41) that the equilibrium path in the $\theta_{R}, w$ plane cannot be steeper than the iso-TFP-growth curves. This must imply that TFP growth first falls. Then consider what happens if $\theta_{R}$ falls over time 
(initial phase). If $\theta_{R}$ never falls below $\theta_{T}$, TFP growth keeps falling until $\theta_{R}$ starts rising (second phase). If, on the other hand, $\theta_{R}$ falls below $\theta_{T}$, TFP growth starts rising before $\theta_{R}$ starts rising. When $\theta_{R}$ starts rising, TFP growth falls until $\theta_{R}$ exceeds $\theta_{T}$ again after which TFP growth increases over time. This proves (iv).

QED

\section{Proof of Proposition 5}

The problem of the social planner is to maximize $\int_{0}^{\infty} \ln \left(C_{Y}\right) e^{-\rho t} d t$ subject to

$$
\begin{array}{ccc}
\dot{Q}_{R}=\frac{D_{R} Q_{R}}{\kappa_{R}}, & \dot{Q}_{L}=\frac{D_{L} Q_{L}}{\kappa_{L}}, & \dot{E}=-(1+\mu) R, \\
C_{Y}=\varphi F\left(Q_{L} L, Q_{R} R\right), & L=L^{S}-D=L^{S}-D_{R}-D_{L}, &
\end{array}
$$

$\varphi$ being the proportion of output spent on consumption. Assuming interior solution and using $\theta_{R}=\frac{\partial F}{\partial\left(Q_{R} R\right)} \frac{Q_{R} R}{Y}$, the first order (Pontryagin Maximum Principle) conditions are

$$
\begin{aligned}
& \frac{\partial H}{\partial D}=-\frac{1}{C_{Y}} \frac{\partial F}{\partial\left(Q_{L} L\right)} Q_{L}+\lambda_{L} \frac{Q_{L}}{\kappa_{L}}=0 \rightarrow \frac{\theta_{L} \kappa_{L}}{\varphi L}=\lambda_{L} Q_{L} \\
& \frac{\partial H}{\partial D_{R}}=\lambda_{R} \frac{Q_{R}}{\kappa_{R}}-\lambda_{L} \frac{Q_{L}}{\kappa_{L}}=0 \quad \text { if } D \in(0, D) \\
& \frac{\partial H}{\partial R}=\frac{1}{C_{Y}} \frac{\partial F}{\partial\left(Q_{R} R\right)} Q_{R}-\lambda_{E}(1+\mu)=0 \rightarrow \frac{\theta_{R}}{\varphi}=R \lambda_{E}(1+\mu) \\
& \frac{\partial H}{\partial Q_{L}}=\frac{1}{C_{Y}} \frac{\partial F}{\partial\left(Q_{L} L\right)} L+\frac{\lambda_{L}\left(D-D_{R}\right)}{\kappa_{L}}=\rho \lambda_{L}-\dot{\lambda}_{L} \rightarrow \frac{\theta_{L}}{\varphi \lambda_{L} Q_{L}}+\widehat{Q}_{L}=\rho-\hat{\lambda}_{L} \\
& \frac{\partial H}{\partial Q_{R}}=\frac{1}{C_{Y}} \frac{\partial F}{\partial\left(Q_{R} R\right)} R+\frac{\lambda_{R} D_{R}}{\kappa_{R}}=\rho \lambda_{R}-\dot{\lambda}_{R} \rightarrow \frac{\theta_{R}}{\varphi \lambda_{R} Q_{R}}+\widehat{Q}_{R}=\rho-\hat{\lambda}_{R} \\
& \frac{\partial H}{\partial E}=0 \rightarrow \hat{\lambda}_{E}=\rho
\end{aligned}
$$

$\lambda_{R}, \lambda_{L}$ and $\lambda_{E}$ being the costate variables. Since, in the long run, $\dot{\theta}_{R}=\dot{\theta}_{L}=\dot{L}=0$, (A.3) implies $\hat{Q}_{L}(\infty)-\hat{\lambda}_{L}(\infty)=0$ and, from (A.4), we also have $\hat{Q}_{R}(\infty)-\hat{\lambda}_{R}(\infty)=0$. Using (A.5) and (A.8) we get $\hat{R}(\infty)=\widehat{Q}_{\mu}(\infty)-\rho$. Combining (A.4), (A.6) and (A.7) and using $\hat{Q}_{L}-\hat{\lambda}_{L}=\hat{Q}_{R}-\hat{\lambda}_{R}=0$ we conclude that $\frac{\theta_{R}(\infty)}{\theta_{L}(\infty)}=\frac{\kappa_{R}}{\kappa_{L}}$ and using $\theta_{R}+\theta_{L}=\kappa_{R}+\kappa_{L}=1$ we get $\theta_{R}(\infty)=\kappa_{R}$. The proposition is proved by comparing this value with that corresponding to the steady-state of the market equilibrium (cf. proof of Proposition 3).

QED. 


\section{Appendix B. The firm's maximization problem: optimality condition (29) and the}

\section{transversality condition}

Intermediate goods firm $k$ in sector $i$ maximizes the present value of its profits, given by $\int e^{-r t}\left(p_{m i k} m_{i k}-q_{i k} m_{i k} p_{y}-w_{D} D_{i k}\right) d t$, subject to (6) and (28) and using $m_{i k}$ and $D_{i k}$ as controls, taking as given all prices, aggregate variables and quantities of other firms. Collecting terms that are beyond the firm's control in $\bar{P}$, we may write (6) as $p_{m i k}=\bar{P} q_{i k} m_{i k}{ }^{1-\beta}$. Then, we may write the current-value Hamiltonian $\left(H_{i k}\right)$ and the first order conditions as

$$
\begin{aligned}
& H_{i k}=\bar{P} q_{i k} m_{i k}^{1-\beta}-q_{i k} m_{i k} p_{Y}-w_{D} D_{i k}+p_{q i k}\left(Q_{i} / \kappa_{i}\right) D_{i k} \\
& (1-\beta) \bar{P} q_{i k} m_{i k}^{-\beta}=q_{i k} p_{Y} \\
& p_{q i k}=w_{D} \kappa_{i} / Q_{i} \\
& \bar{P} m_{i k}^{1-\beta}-m_{i k} p_{Y}=r p_{q i k}-\dot{p}_{q i k}
\end{aligned}
$$

Eliminating $\bar{P}=p_{m i k} / q_{i k} m_{i k}{ }^{1-\beta}$ in (B.2) we find (7). From (B.3) we get $\hat{p}_{q i k}=\hat{w}_{D}-\hat{Q}_{i}$ . Using this equation together with (B.3) and (B.4) to eliminate $p_{q i k}$ and $\hat{p}_{q i k}$, we get (29). The transversality condition reads $\lim _{t \rightarrow \infty} p_{q i k} q_{i k} e^{-r t}=0$. Integrating over all firms in sector $i$, using (9), and eliminating $p_{q i k}$ with (B.3), we find:

$$
\lim _{t \rightarrow \infty} \int_{0}^{1} p_{q i k} q_{i k} e^{-r t} d k=\lim _{t \rightarrow \infty} \frac{w_{D} \kappa_{i}}{Q_{i}} e^{-r t} \int_{0}^{1} q_{i k} d k=w_{D} \kappa_{i} \lim _{t \rightarrow \infty} e^{-r t}=0
$$

Hence, a necessary condition for the transversality condition to hold is $\lim _{t \rightarrow \infty} r(t)-\hat{w}_{D}(t) \geq 0$. 
Figure 1 US energy share.

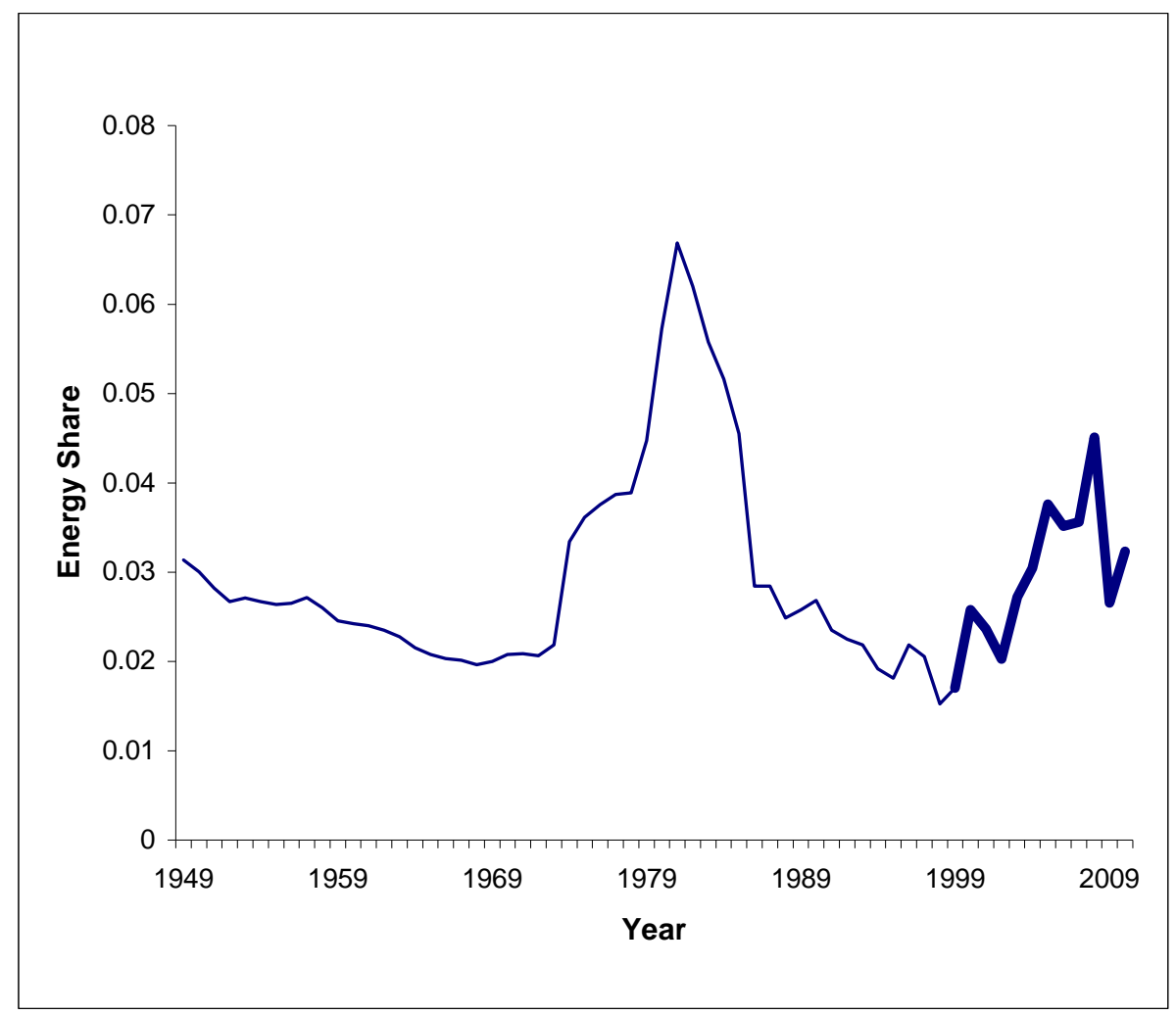

Calculated as total energy expenditure (energy consumption times energy price) over GDP. Data from "Annual Energy Review" from the US Energy Information Administration. The thin line replicates Jones (2002, Table 9.3). The thick line is our update (using the same source as Jones). 
Figure 2 Phase diagram (endogenous technological change)

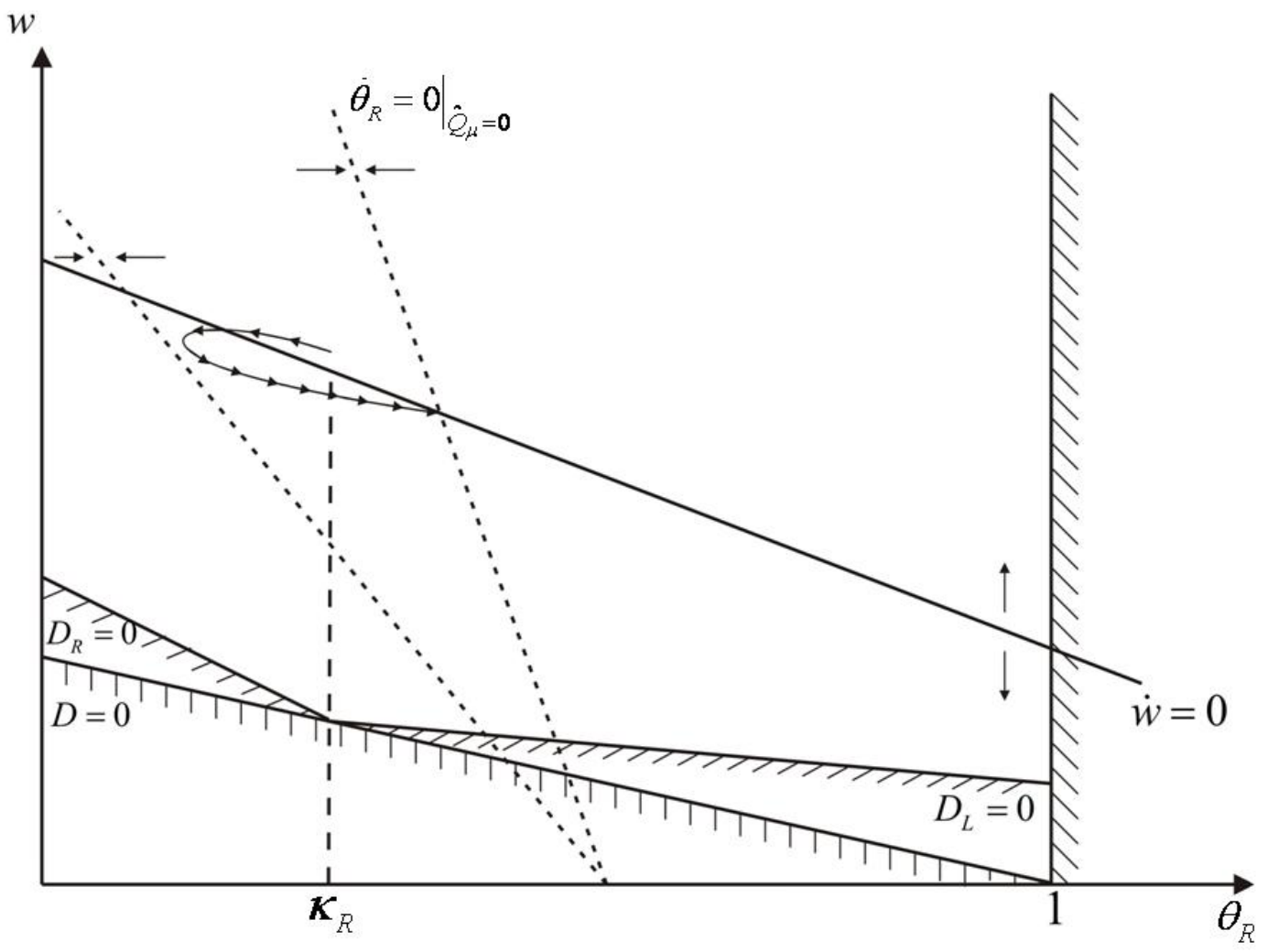


Figure 3 Energy share (observed and smoothed version)

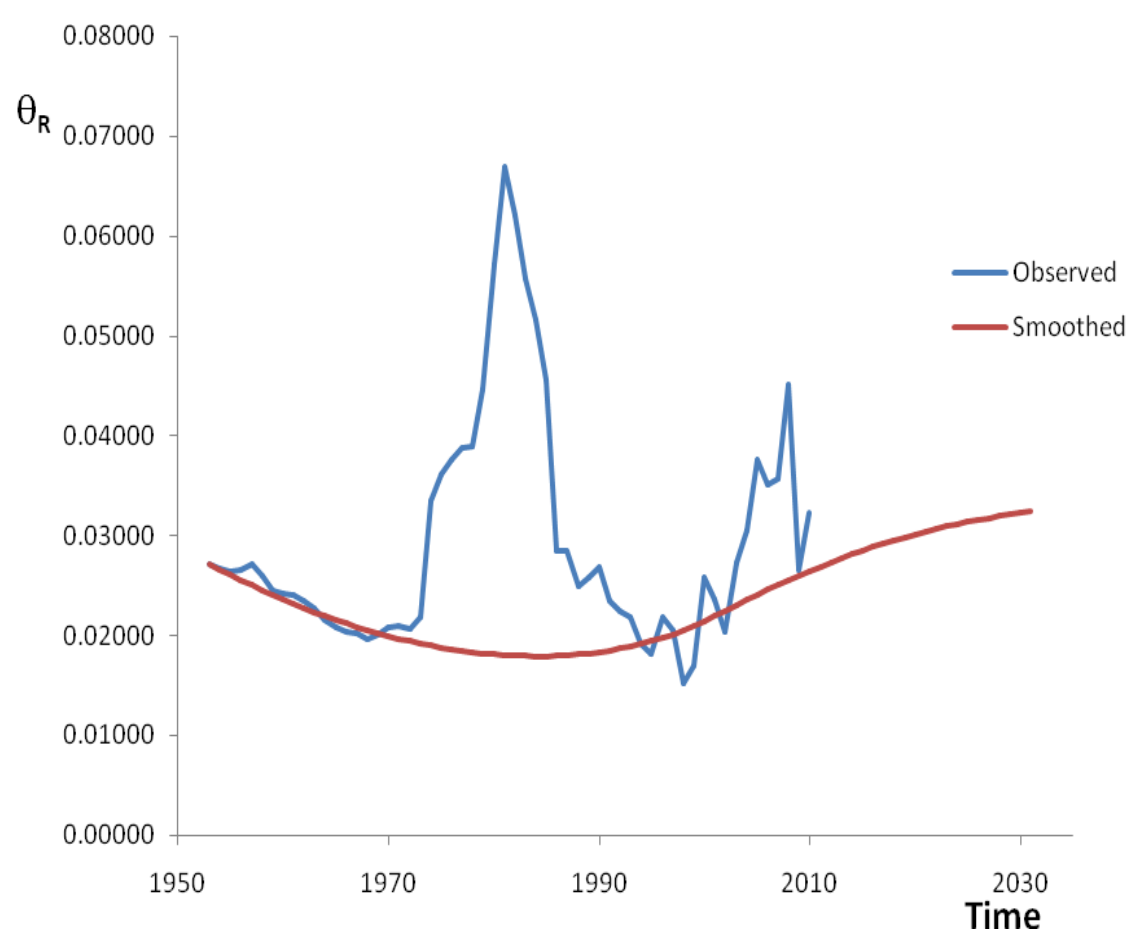


Figure 4: Improvements in mining efficiency

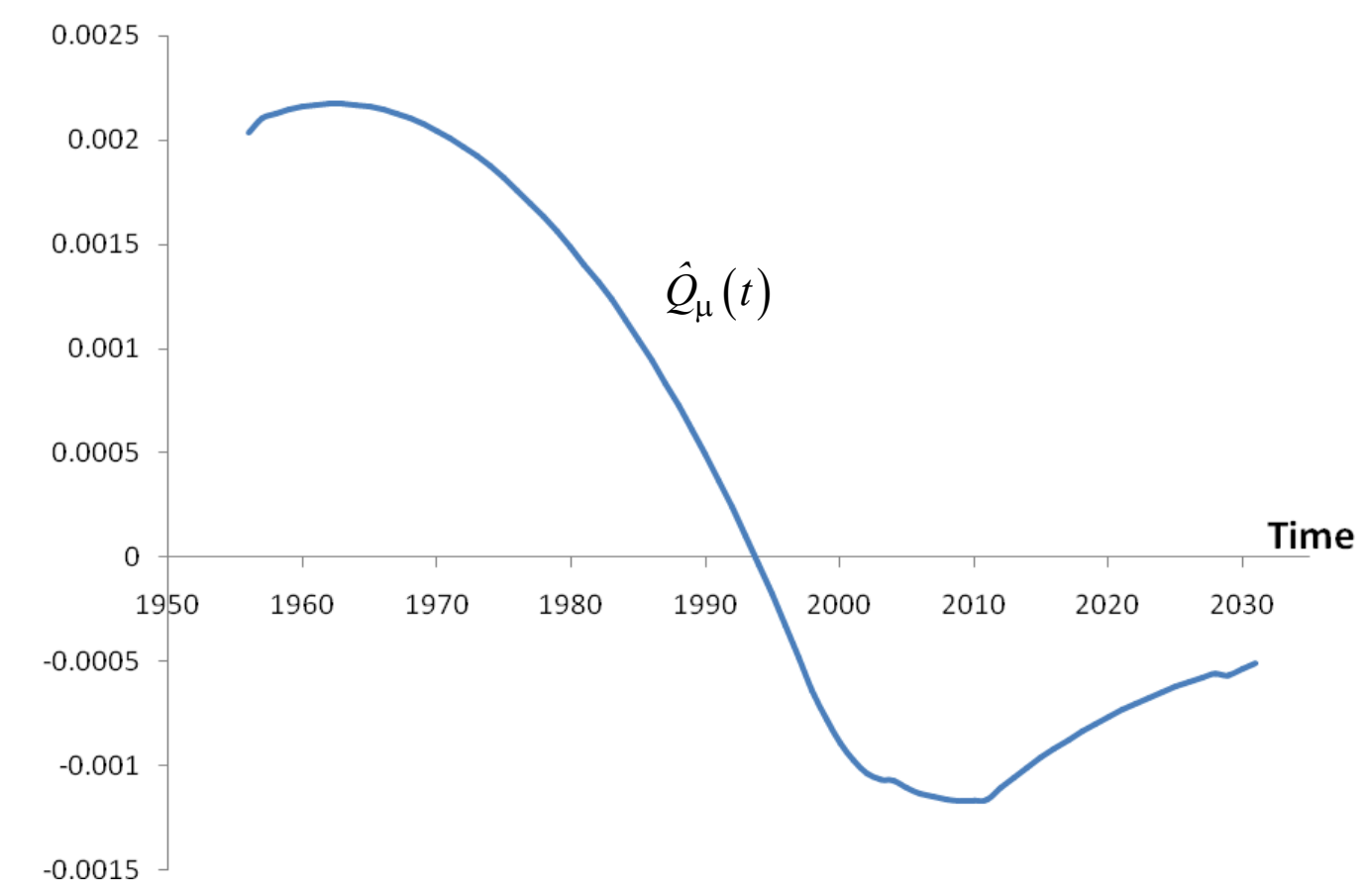

Constructed to the criterion or obtaning as a result the smoothed version of the energy share 
Figure 5: Energy per capita, energy share and relative energy price (observed data)

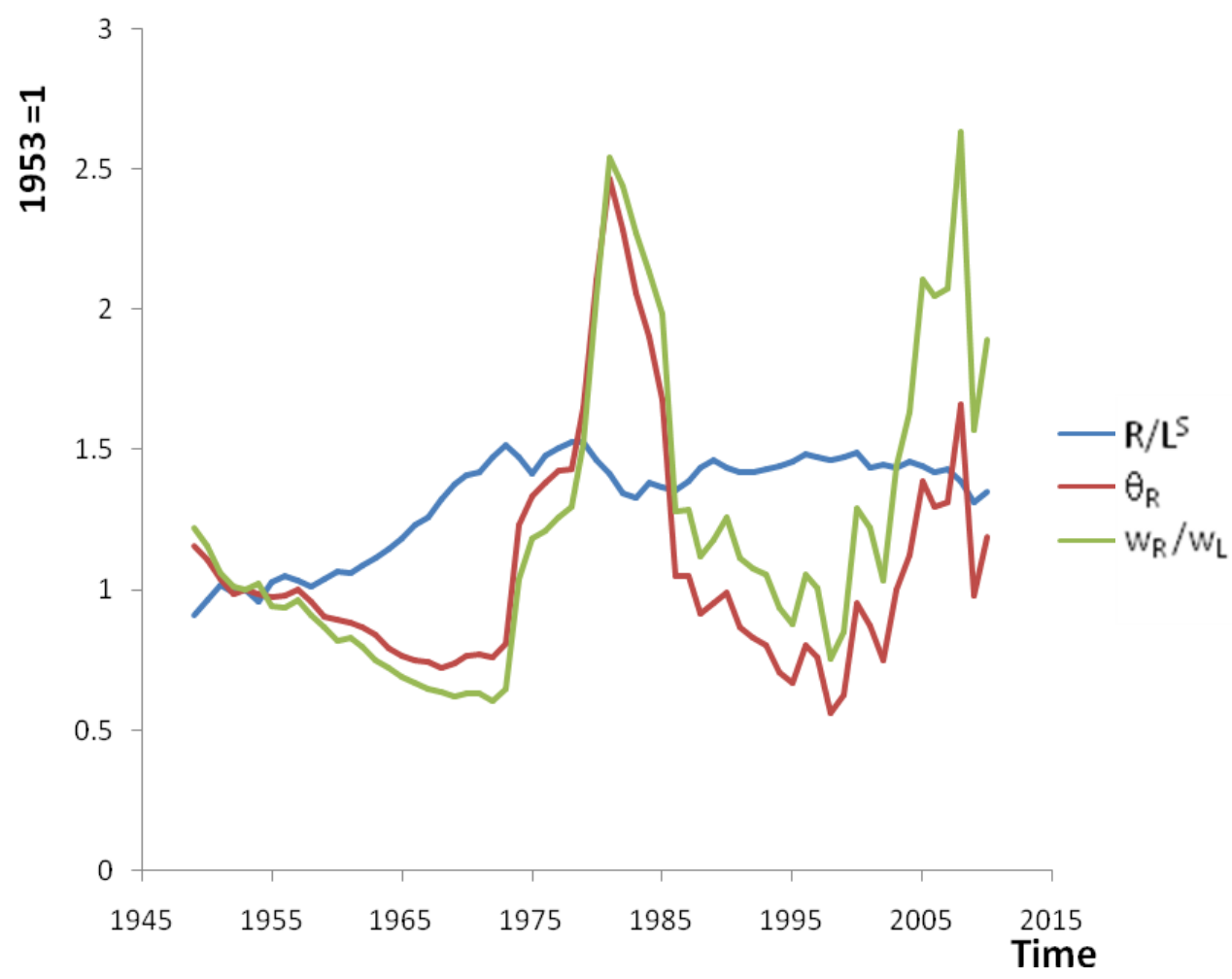


Figure 6: Energy per capita, energy share and relative energy price (generated by the model)

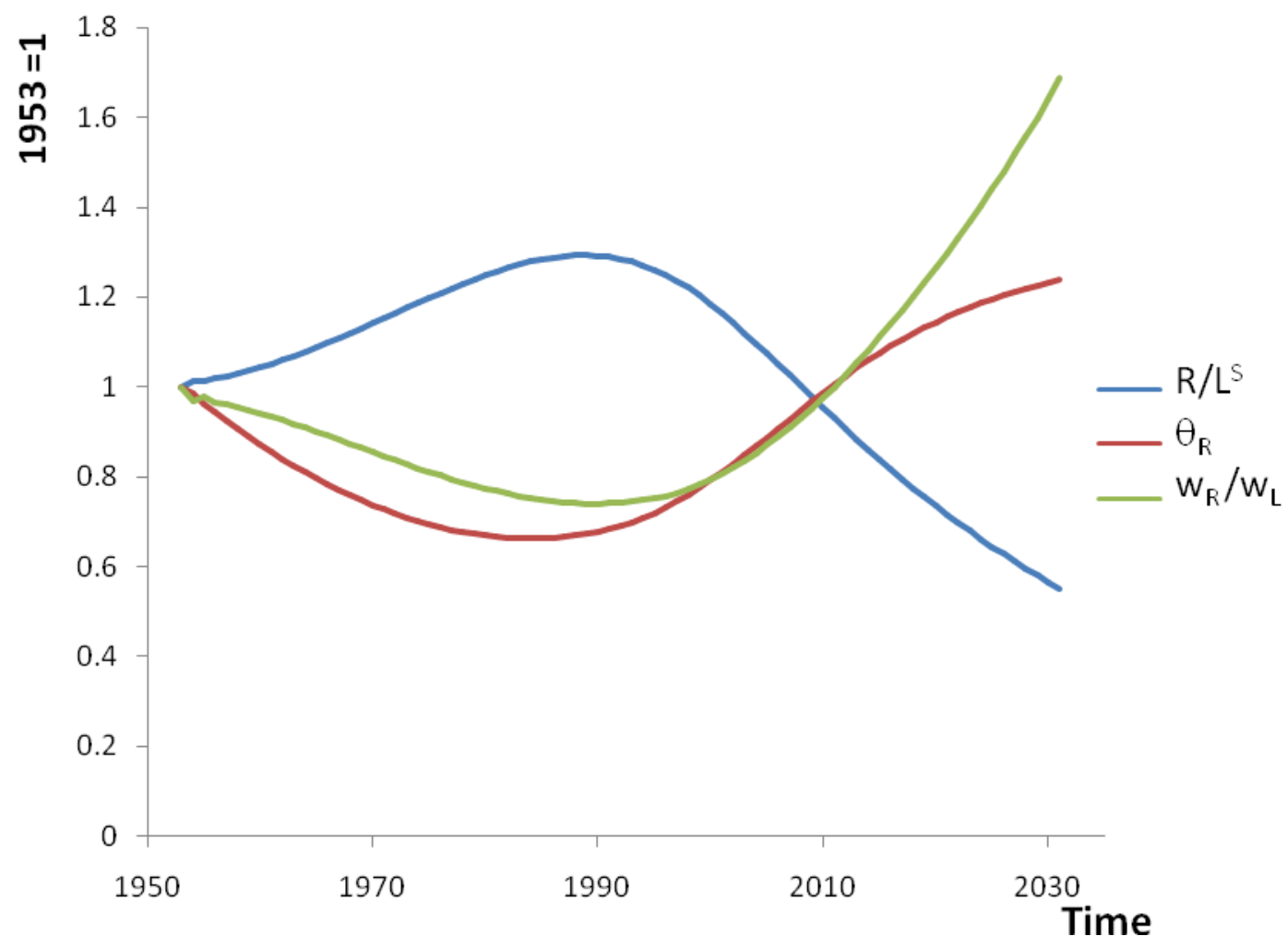

